\title{
Non-Abelian chiral spin liquid in a quantum antiferromagnet revealed by an iPEPS study
}

\author{
Ji-Yao Chen, ${ }^{1}$ Laurens Vanderstraeten, ${ }^{2}$ Sylvain Capponi, ${ }^{1}$ and Didier Poilblanc ${ }^{1,3}$ \\ ${ }^{1}$ Laboratoire de Physique Théorique, IRSAMC, Université de Toulouse, CNRS, UPS, 31062 Toulouse, France \\ ${ }^{2}$ Department of Physics and Astronomy, University of Ghent, Krijgslaan 281, 9000 Gent, Belgium \\ ${ }^{3}$ Institute of Theoretical Physics, École Polytechnique Fédérale de Lausanne (EPFL), CH-1015 Lausanne, Switzerland
}

(Received 19 July 2018; published 8 November 2018)

\begin{abstract}
Abelian and non-Abelian topological phases exhibiting protected chiral edge modes are ubiquitous in the realm of the fractional quantum Hall $(\mathrm{FQH})$ effect. Here, we investigate a spin-1 Hamiltonian on the square lattice which could, potentially, host the spin liquid analog of the (bosonic) non-Abelian Moore-Read FQH state, as suggested by exact diagonalization of small clusters. Using families of fully SU(2)-spin symmetric and translationally invariant chiral projected entangled pair states (PEPS), variational energy optimization is performed using infinite-PEPS methods, providing good agreement with density matrix renormalization group (DMRG) results. A careful analysis of the bulk spin-spin and dimer-dimer correlation functions in the optimized spin liquid suggests that they exhibit long-range "gossamer tails". From the investigation of the entanglement spectrum, we observe sharply defined chiral edge modes following the prediction of the $S U(2)_{2}$ Wess-ZuminoWitten theory and exhibiting a conformal field theory (CFT) central charge $c=3 / 2$, as expected for a MooreRead chiral spin liquid. Using the PEPS bulk-edge correspondence, we argue the "weak" criticality of the bulk is in fact a finite- $D$ artifact of the chiral PEPS, which quickly becomes (practically) irrelevant as the PEPS bond dimension $D$ is increased. We conclude that the PEPS formalism offers an unbiased and efficient method to investigate non-Abelian chiral spin liquids in quantum antiferromagnets.
\end{abstract}

DOI: 10.1103/PhysRevB.98.184409

\section{INTRODUCTION AND MODEL}

The two-dimensional (2D) electron gas experiencing longrange Coulomb repulsion and subject to a strong magnetic field-hence breaking time-reversal (TR) symmetry—can exhibit plethora of topological fractional quantum Hall $(\mathrm{FQH})$ phases at simple rational filling fractions $v[1]$. FQH states are characterized by topological order - the ground state (GS) degeneracy depends on the system topology [2,3]— and by chiral edge modes localized at the system boundaries (if any) and propagating in one direction only $[4,5]$. Such edge modes are gapless and described by known $(1+1)$-dimensional conformal field theories (CFT). The bulk excitations of the FQH states are fractionalized anyons [6] which could have either Abelian statistics, as in the Laughlin state [7], or nonAbelian statistics [8,9], as in the Moore-Read (MR) state [10]. Non-Abelian $\mathrm{SU}(2)_{k}$ anyons (for $k>1$ ) are described by well-known deformations of $\mathrm{SU}(2)$, in which only the first $k+1$ angular momenta $j=0, \frac{1}{2}, 1, \ldots, \frac{k}{2}$ of $\mathrm{SU}(2)$ occur. The MR state harbors $j=\frac{1}{2}$ Ising anyons (realized for $k=$ 2 ), descendants of vortices in (two-dimensional) $p+i p$ superconductors [11,12], and exhibiting simple fusion rules, $\frac{1}{2} \times \frac{1}{2} \rightarrow 0+1$.

Fractional Chern insulators (FCI) [13,14] offer the most direct implementation of the $\mathrm{FQH}$ physics on the lattice, still requiring a (gauge) magnetic field to generate electronic bands with nontrivial topological properties (i.e., nonzero Chern numbers), and strong (local) interactions. In the case of Mott insulators, such as those realizing quantum magnets, the appropriate setting to realize $\mathrm{FQH}$ physics is less clear. It is well known, nevertheless, since the pioneering work of Kalmeyer and Laughlin (KL) [15], that simple $\mathrm{FQH}$ wavefunctions (such as the Abelian bosonic $v=1 / 2$ Laughlin state) can be "localized" on the sites of a 2D lattice in order to realize chiral (singlet) spin liquids (CSL) [16], spin analogs of the parent $\mathrm{FQH}$ states. However, it is largely unknown whether and under which conditions simple local Hamiltonians describing (frustrated) quantum antiferromagnets can host such spin liquids, in particular the non-Abelian ones. Recent numerical investigations of a spin- $1 / 2$ chiral Heisenberg antiferromagnetic model (HAFM) on the kagomé lattice $[17,18]$ suggest that a scalar chiral interaction on all triangular units can indeed stabilize a spin liquid of the $v=1 / 2 \mathrm{KL}$ type. Similar Abelian CSL were also uncovered in spin- $1 / 2$ chiral antiferromagnets on the triangular lattice $[19,20]$. Interestingly, the CSL can also emerge in spin-1/2 time-reversal invariant frustrated magnets [18,21]. Kitaev's anisotropic honeycomb model in the presence of an external magnetic field [22] is, so far, the only indisputable example of a local (lattice) Hamiltonian hosting a non-Abelian CSL, but local spin-1 Hamiltonians on triangular and kagome lattices have also been proposed [23,24], as well as coupled-wire constructions of $\mathrm{SU}(2)_{k}$ CSL [25]. A definite identification of local SU(2)invariant models realizing non-Abelian CSL is therefore needed and the goal of this study.

Further progress in the field of chiral SL have been launched by the constructions of parent quantum spin Hamiltonians [26-29] designed to host various spin analogs of the $\mathrm{FQH}$ liquids. For example, by rewriting KL-like states as correlators of CFT primary fields, a systematic construction of parent Hamiltonians can be obtained. It turns out that, generically, the obtained parent Hamiltonians show long-range 
(algebraic) interactions. For example, SU(2)-invariant spin$1 / 2$ and spin-1 Hamiltonians with long-range three site interactions [like $\mathbf{S}_{i} \cdot\left(\mathbf{S}_{j} \times \mathbf{S}_{k}\right)$ ] have been found to realize the bosonic (Abelian) Laughlin and (non-Abelian) Moore-Read FQH phases, respectively $[28,30]$. Using such a construction, it was also shown that the spin-1/2 KL spin liquid exhibits the expected chiral edge states [31]. Furthermore, it was argued that local chiral antiferromagnetic Heisenberg models based on some truncation and fine-tuning of the parent Hamiltonians also host the same topological Abelian [32,33] and non-Abelian [30] phases. Similar to the non-Abelian Kitaev's phase on the hexagonal lattice [22,34], the spin-1 non-Abelian CSL is expected to host Ising anyons in the bulk. However, the proposed spin-1 local chiral HAFM is quite far from the initial parent Hamiltonian and its detailed investigation is called for.

Besides KL and CFT constructions, topological chiral spin liquids can also be designed using the framework of projected entangled pair states (PEPS) [35-39], a class of 2D tensor networks [40]. Generally, topological order can be easily implemented in PEPS from local gauge symmetries [41]. The simplest chiral PEPS is based on a chiral extension $[42,43]$ of the spin-1/2 resonating valence bond (RVB) state [44-46], originally defined by Anderson as an equal-weight superposition of valence bond configurations [47]. Such a simple PEPS turned out to be critical although, surprisingly, exhibiting well-defined chiral edge modes consistent with the $\mathrm{SU}(2)_{1}$ Wess-Zumino-Witten (WZW) CFT of central charge $c=1$. A more general and systematic construction of PEPS chiral (and nonchiral [48]) spin liquids has been made recently possible, thanks to a general classification of SU(2) and translationally invariant PEPS according to their symmetry properties under point group operations [49]. Combining this classification with a corner transfer matrix renormalization group (CTMRG) algorithm [50], one of us investigated the physics of the simple spin-1/2 chiral HAFM mentioned above [51]. Topological order was identified from sharply defined chiral edge modes but, surprisingly, numerical results suggested critical correlations in the bulk, as for the simpler chiral RVB analog. Whether this feature is a generic property of chiral PEPS [52] is not clear so far. Investigation of new chiral HAFM using PEPS methods is therefore necessary.

Here, we shall consider the spin-1 chiral HAFM defined on the two-dimensional square lattice, as introduced in Ref. [30]:

$$
\begin{aligned}
H= & J_{1} \sum_{\langle i, j\rangle} \mathbf{S}_{i} \cdot \mathbf{S}_{j}+J_{2} \sum_{\langle\langle k, l\rangle\rangle} \mathbf{S}_{k} \cdot \mathbf{S}_{l} \\
& +K_{1} \sum_{\langle i, j\rangle}\left(\mathbf{S}_{i} \cdot \mathbf{S}_{j}\right)^{2}+K_{2} \sum_{\langle\langle k, l\rangle\rangle}\left(\mathbf{S}_{k} \cdot \mathbf{S}_{l}\right)^{2} \\
& +K_{c} \sum_{\square}\left[\mathbf{S}_{i} \cdot\left(\mathbf{S}_{j} \times \mathbf{S}_{k}\right)+\mathbf{S}_{j} \cdot\left(\mathbf{S}_{k} \times \mathbf{S}_{m}\right)\right. \\
& \left.+\mathbf{S}_{i} \cdot\left(\mathbf{S}_{j} \times \mathbf{S}_{m}\right)+\mathbf{S}_{i} \cdot\left(\mathbf{S}_{k} \times \mathbf{S}_{m}\right)\right],
\end{aligned}
$$

where the first and third sums are taken over nearest-neighbor (NN) bonds and the second and fourth sums run over nextnearest-neighbor (NNN) bonds. The chiral term of amplitude $K_{c}$ is defined on every plaquette of four sites $(i, j, k, m)$ ordered in (let say) anticlockwise direction. The parameters entering (1) have been obtained by a careful fine tuning, optimizing the overlap of the exact GS on small finite-size clusters with the lattice CFT correlator describing the non-Abelian Moore-Read FQH state (on the lattice) [30]. We will here adopt these fine-tuned parameters (retaining only 3 digits), $J_{1}=1, J_{2}=0.623, K_{1}=-0.176, K_{2}=0.323$, and $K_{c}=$ 0.464 . Note that the related spin- $1 / 2$ chiral HAFM introduced in Ref. [28] and studied in Ref. [51] contains only the $J_{1}$ and $J_{2}$ bilinear terms and the $K_{c}$ chiral term since the biquadratic interactions $K_{1}$ and $K_{2}$ become irrelevant for spin-1/2.

In order to explore the physics of the above model, we combine different numerical techniques such as Lanczos exact diagonalizations (ED), density matrix renormalization group (DMRG) [53] and tensor network methods [35-39], all reviewed in Sec. II. In particular, we shall focus on spin-1 $\mathrm{SU}(2)$-symmetric PEPS to describe the chiral spin liquid phase. More precisely, we construct (disconnected) families of PEPS breaking time-reversal $(\mathrm{T})$ and parity $(\mathrm{P})$ symmetrieswithout breaking $\mathrm{PT}$ - providing a faithful representation of chiral spin liquids directly in the thermodynamic limit. In contrast to usual PEPS calculations, which approach the ground state of the model via imaginary time evolution (and could get trapped in local minima), we use a more elegant and secure framework based on a variational optimization scheme (combined with a CTMRG algorithm), taking advantage of the reduced number of variational parameters in the fully symmetric Ansatz.

Using such state-of-the-art numerical techniques, we shall address a number of important issues. First, in Sec. III, we shall investigate the property of the bulk system, i.e., whether it exhibits short-range correlations like its "parent" FQH Moore-Read state or whether it is critical such as the spin-1/2 chiral PEPS analog. Second, in Sec. IV, we shall consider the edge spectrum, seeking to characterize topological chiral order, and looking for evidence of its non-Abelian character. Finally, we shall discuss the results in the last section, and make some conjecture. Experimental setups will also be briefly discussed.

\section{SHORT SUMMARY OF NUMERICAL METHODS}

\section{A. Lanczos exact diagonalizations}

In Refs. [30], the parameters of the spin-1 model have been obtained using exact diagonalization of small lattices (up to $4 \times 4$ ) on the plane or on the cylinder, fine tuning the overlap of the exact GS with the targeted non-Abelian chiral state. Here we diagonalize, using Lanczos ED methods, $4 \times 4$ and $\sqrt{20} \times \sqrt{20}$ square tori-exhibiting the full translation and (at least) $C_{4}$ point group symmetries of the square lattice-to investigate the low-energy spectrum of the model. In contrast to the planar geometry, in the case of a torus geometry, the GS is expected to become degenerate (threefold degenerate for the Moore-Read state) in a gapped topological phase and in the limit of very large sizes. Hence fundamental differences from the previous computations are expected, even on small clusters.

\section{B. Density matrix renormalization group}

A standard approach for matrix product state (MPS) simulations of 2D systems consists in studying cylinders (with $N=L \times W$ sites, $L>W$ ) with periodic (respectively, open) boundary conditions in the short (respectively, long) 
dimension. We have used the ITENSOR library for these calculations, ${ }^{1}$ in particular using total $S_{z}$ conservation. We have observed that convergence is very hard to achieve for $W>8$ due to the large entanglement entropy of a half-system and the maximum number of states kept in the simulation was $m=2000$. This may be related to criticality of the system (see later) or large correlation length. Therefore we have used MPS computations as a way to extract the ground-state energy per site $e_{0}(L, W)$. First, for a given system size $L \times W$, we can extrapolate the total energy versus discarded weight. Then, at fixed width $W$, we can estimate $e_{0}(W)$ by extrapolating $e_{0}(L, W)$ versus $1 / L$. Another estimate of the same quantity can be obtained from different cylinders of width $W$ and lengths $L_{1}$ and $L_{2}$, simply by subtracting total energies $E_{0}$ :

$$
e_{0}(W)=\frac{E_{0}\left(L_{2}, W\right)-E_{0}\left(L_{1}, W\right)}{L_{2}-L_{1}}
$$

which has the advantage to reduce finite-size effects due to the open boundaries on the edges. At last, we can extrapolate $e_{0}(W)$ versus $1 / W$ as shown in Sec. III B.

Quite interestingly, we can also obtain an exact upper bound using cylinders with open boundary conditions (OBC) in both directions, and using their ground-state wave functions as a variational one for the infinite system [54]. These values are also reported in Sec. III B.

\section{C. iPEPS method}

\section{Singlet chiral PEPS Ansatz}

Our infinite-PEPS (iPEPS) method-directly in the thermodynamic limit-relies first on the construction of generic PEPS Ansätze fulfilling all the necessary symmetry properties of the targeted chiral spin liquid. First, for convenience, we apply a $\pi$ rotation along the $Y$ spin axis on all sites of one of the two sublattices, which enables to express the (approximate) GS wave function(s) in terms of a unique site tensor $\mathcal{A}_{\alpha \beta \gamma \delta}^{s}$ (instead of two, one for each sublattice), where the greek indices label the states of the $D$-dimensional virtual space $\mathcal{V}$ attached to each site in the $z=4$ directions of the lattice, and $s=0, \pm 1$ is the $S_{z}$ component of the physical spin 1. A chiral spin liquid bears symmetry properties that greatly constrain the PEPS Ansatz. To construct such an Ansatz, we use a general classification of all (translationally invariant) $\mathrm{SU}(2)$-symmetric (i.e., invariant under any spin rotation) PEPS proposed recently [49], in terms of the five irreducible representations (IRREPs) $A_{1}, A_{2}, B_{1}, B_{2}$, and $E$ of the square lattice $C_{4 v}$ point group [55]. In this setting, the virtual space $\mathcal{V}$ is defined as any direct sum of SU(2) IRREPs. Since the chiral spin liquid only breaks $\mathrm{P}$ and $\mathrm{T}$ but does not break the product PT, the simplest adequate PEPS complex tensors have the following forms:

$$
\begin{gathered}
\mathcal{A}=\mathcal{A}_{1}+i \mathcal{A}_{2}=\sum_{a=1}^{N_{1}} \lambda_{a}^{1} \mathcal{A}_{1}^{a}+i \sum_{b=1}^{N_{2}} \lambda_{b}^{2} \mathcal{A}_{2}^{b}, \\
\mathcal{B}=\mathcal{B}_{1}+i \mathcal{B}_{2}=\sum_{a=1}^{M_{1}} \mu_{a}^{1} \mathcal{B}_{1}^{a}+i \sum_{b=1}^{M_{2}} \mu_{b}^{2} \mathcal{B}_{2}^{b},
\end{gathered}
$$

\footnotetext{
${ }^{1}$ ITENSOR C ++ library, available at http://itensor.org.
}

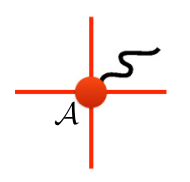

(a)

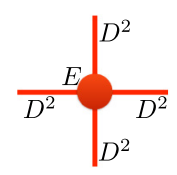

(b)

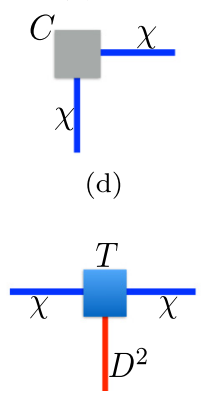

(e)

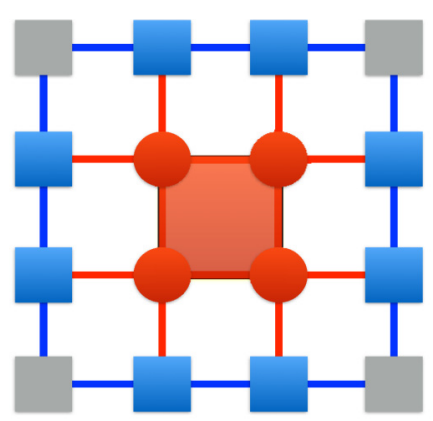

(c)

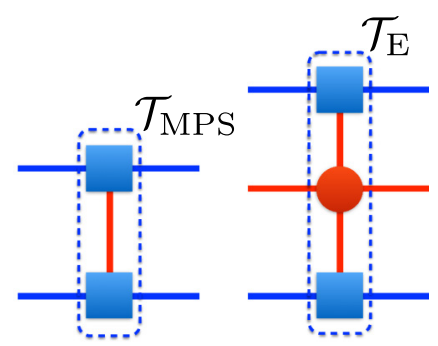

(g)
FIG. 1. CTMRG for the chiral PEPS with one-site unit cell. (a) $A_{1}+i A_{2}$ PEPS tensor $\mathcal{A}$. (b) Double tensor $E$ obtained by tracing out physical indices $E=\sum_{s} \overline{\mathcal{A}}^{s} \mathcal{A}^{s}$ (or $E=\sum_{s} \overline{\mathcal{B}}^{s} \mathcal{B}^{s}$ ), where $\overline{\mathcal{A}}^{s}$ $\left(\overline{\mathcal{B}}^{s}\right)$ is the complex conjugate of $\mathcal{A}^{s}\left(\mathcal{B}^{s}\right)$. (c) CTMRG environment for $2 \times 2$ cluster, constructed from the (real) corner matrix $C$ and the (hermitian) boundary tensor $T$, depicted separately in (d) and (e), respectively. The environment bond dimension is chosen to be $\chi=$ $k D^{2}\left(k \in \mathbb{N}_{+}\right)$. The energy density is calculated by inserting either the identity operator or the local hamiltonian operator in the red shaded $2 \times 2$ cluster. (f) and (g) are the transfer matrices constructed from the $T$ tensor only, and from the $T$ and $E$ tensors, respectively. The maximal correlation length can be obtained from the largest two eigenvalues of both transfer matrices (see text).

graphically shown in Fig. 1(a), where the real elementary tensors $\mathcal{A}_{1}^{a}\left(\mathcal{B}_{1}^{a}\right)$ and $\mathcal{A}_{2}^{b}\left(\mathcal{B}_{2}^{a}\right)$ have the same set of virtual spins and transform according to the $A_{1}\left(B_{1}\right)$ and $A_{2}\left(B_{2}\right)$ IRREP of the point group $C_{4 v}$ of the square lattice. $N_{1}$ and $N_{2}\left(M_{1}\right.$ and $\left.M_{2}\right)$ are the numbers of such elementary tensors in each class, respectively. $\lambda_{a}^{1}$ and $\lambda_{a}^{2}\left(\mu_{b}^{1}\right.$ and $\left.\mu_{b}^{2}\right)$ are arbitrary real coefficients of these tensors. Note that, in the atomic orbital language, $\mathcal{A}$ and $\mathcal{B}$ would correspond to $s+i g$ and $d_{x^{2}-y^{2}}+i d_{x y}$ orbitals, respectively. The PEPS wave function is obtained by the contraction of all site tensors (i.e., by summing all virtual indices on the links).

The symmetric tensors up to $D \leqslant 6$ have been tabulated in Ref. [49], and their numbers $\mathcal{D}_{A}=N_{1}+N_{2}$ and $\mathcal{D}_{B}=$ $M_{1}+M_{2}$, for the most relevant virtual spaces $\mathcal{V}$, are listed in Table I. For each choice of the virtual space, Eqs. (3) and (4) then provide two families of PEPS. From the table of characters of the $C_{4 v}$ IRREPs, it is clear that $\mathcal{A} \rightarrow \overline{\mathcal{A}}$ and $\mathcal{B} \rightarrow \pm \overline{\mathcal{B}}$ under any of the point group reflections, $R_{x}$ and $R_{y}$ along the crystallographic axes, and $R_{x+y}$ and $R_{x-y}$ along the $\pm 45^{\circ}$ directions. Hence the corresponding PEPS wave function transforms into its complex conjugate, which is equivalent (for a singlet state) to the effect of applying time reversal symmetry. 
TABLE I. Numbers of independent SU(2)-symmetric spin-1 tensors for the different virtual spaces we consider, $D \leqslant 6$. The third (fourth) column gives the number of $\mathcal{A}_{1} / \mathcal{B}_{1}\left(\mathcal{A}_{2} / \mathcal{B}_{2}\right)$ tensors and the last column contains the total numbers $\mathcal{D}_{A} / \mathcal{D}_{B}$ of tensors entering Eq. (3)/Eq. (4). $*$ means the states of the PEPS family are all real (i.e., nonchiral).

\begin{tabular}{lcccc}
\hline \hline $\mathcal{V}$ & $D$ & $N_{1} / M_{1}$ & $N_{2} / M_{2}$ & $\mathcal{D}_{A} / \mathcal{D}_{B}$ \\
\hline$\frac{1}{2} \oplus 0$ & 3 & $2 / 2$ & $0 / 1$ & $2^{*} / 3^{*}$ \\
$\frac{1}{2} \oplus \frac{1}{2}$ & 4 & $6 / 9$ & $4 / 3$ & $10 / 12$ \\
$1 \oplus 0$ & 4 & $3 / 5$ & $3 / 1$ & $6 / 6$ \\
$\frac{1}{2} \oplus \frac{1}{2} \oplus 0$ & 5 & $12 / 13$ & $5 / 6$ & $17 / 19$ \\
$1 \oplus \frac{1}{2}$ & 5 & $5 / 5$ & $3 / 4$ & $8 / 9$ \\
$1 \oplus \frac{1}{2} \oplus 0$ & 6 & $13 / 13$ & $8 / 9$ & $21 / 22$ \\
\hline \hline
\end{tabular}

Our chiral PEPS also exhibit a very important gauge symmetry encoded at the level of the local $\mathcal{A}$ and $\mathcal{B}$ tensors. More precisely, the number of spins $1 / 2$ (or half-integer spins, in general) present in the set of virtual degrees of freedom attached to each site is always even. The $\mathbb{Z}_{2}$ gauge symmetry linked to this parity conservation is at the origin of the topological order sustained by the PEPS [41].

\section{CTMRG algorithm}

Once PEPS families have been constructed, the second step is to optimize the Hamiltonian energy with respect to the tensor parameters, for each class separately. The reduced number of parameters (obtained thanks to the implementation of the full state symmetries) allows to perform a "brute force" optimization (in contrast to different variational optimization schemes [56,57]). For each set of PEPS parameters, one then needs to compute the corresponding variational energy, in order to "feed" an efficient minimization routine, i.e., one based on a conjugate gradient (CG) method. The variational energy computation is done directly in the thermodynamic limit using the CTMRG algorithm [50]. After constructing the doublelayer tensor $E$ of Fig. 1(b), one obtains, using a real-space RG method, the environment of Fig. 1(c) surrounding the active $2 \times 2$ region and involving the CTM $C$ and $T$ tensors shown in Figs. 1(d) and 1(e). The identity matrix or the Hamiltonian is then inserted in the active region (between the two layers) to compute the energy per site. The gradient of energy density is then computed by a finite difference method, which is applicable thanks to the small number of parameters. With the energy and its gradient, the CG method can now be used to find the best parameters. Note that, for our chiral PEPS, all $C$ and $T$ tensors in Fig. 1(c) are identical by symmetry (and the $C$ matrix remains Hermitian after each CTMRG step), which simplifies significantly the CTMRG procedure.

\section{Uniform MPS method}

An alternative method for computing effective environments for PEPS in the thermodynamic limit relies on uniform matrix product states (MPS). A transfer matrix is constructed by repeating the double-layer tensor $E$ [Fig. 1(b)] on an infinite linear chain, and we find the transfer-matrix fixed point as a uniform MPS using variational optimization [58]. After
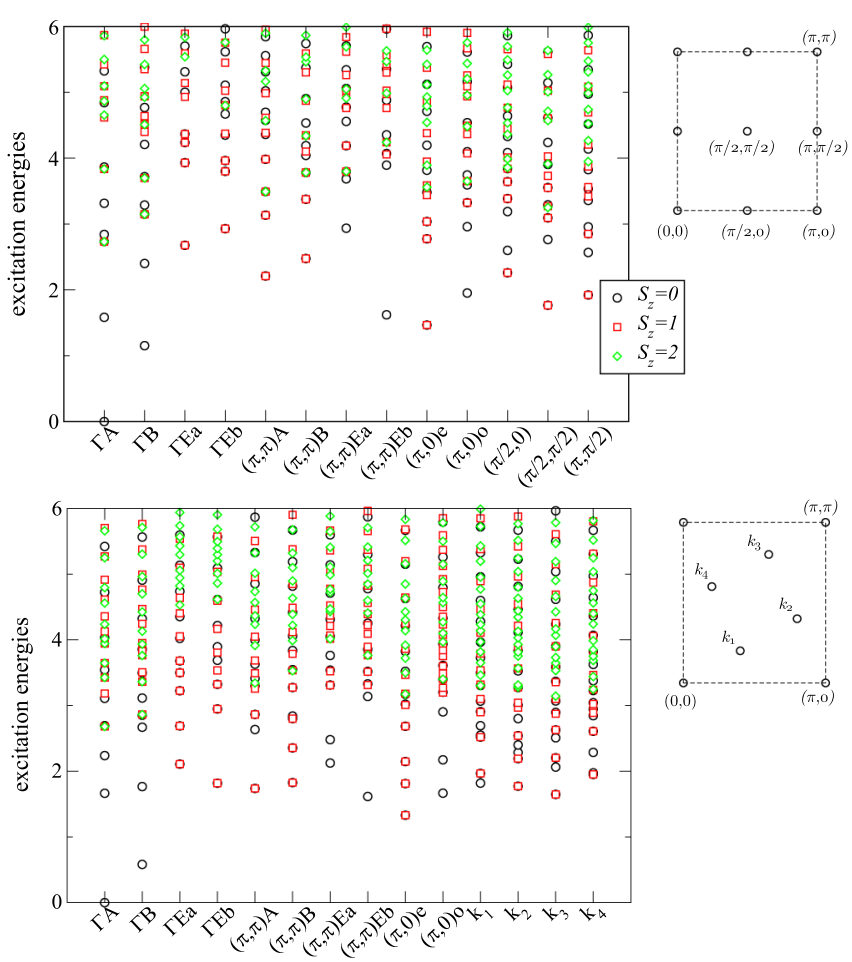

FIG. 2. Lanczos ED of the spin-1 chiral HAFM on a $4 \times 4$ (16-site) (a) and $\sqrt{20} \times \sqrt{20}$ (20-site) (b) tori. The various columns correspond to different IRREPs of the space group and different symbols are used to distinguish eigenstates with different (total) spin quantum numbers. Momenta corresponding to the respective Brillouin zones are shown on the right. The GS energy has been subtracted for clarity.

repeating this procedure in different lattice directions, we find effective environments and we can compute observables from the PEPS. Additionally, the use of channel environments [59] allows to compute correlation functions directly in momentum space.

\section{RESULTS ON BULK PROPERTIES}

\section{A. Low-energy spectra on small tori}

Let us first investigate the spin-1 chiral HAFM on small 16-site and 20-site clusters with periodic boundary conditions and full (or partial) point group symmetry, enabling to $a$ priori block-diagonalize the Hamiltonian matrix according to the irreducible representations (IRREPs) of the cluster space group. We also use the total $S_{z}$ quantum number, enabling to reconstruct the exact $S U(2)$ multiplet structure of the energy spectrum.

The low-energy spectra, split in the various IRREPs, are shown in Figs. 2(a) and 2(b). For the Moore-Read state, we expect to observe three quasidegenerate eigenstates on a torus. In particular, their momentum quantum numbers can be obtained from a simple counting rules [60-62] using partitions $(2,0,2,0, \ldots),(0,2,0,2, \ldots)$, and $(1,1,1,1, \ldots)$, from which we predict that these three states should be at the $\Gamma$ point $[K=(0,0)]$ for 16 - and 20 -site square clusters. However, no clear energy gap separating a group of quasidegenerate singlets from the rest of the spectrum - the signature 


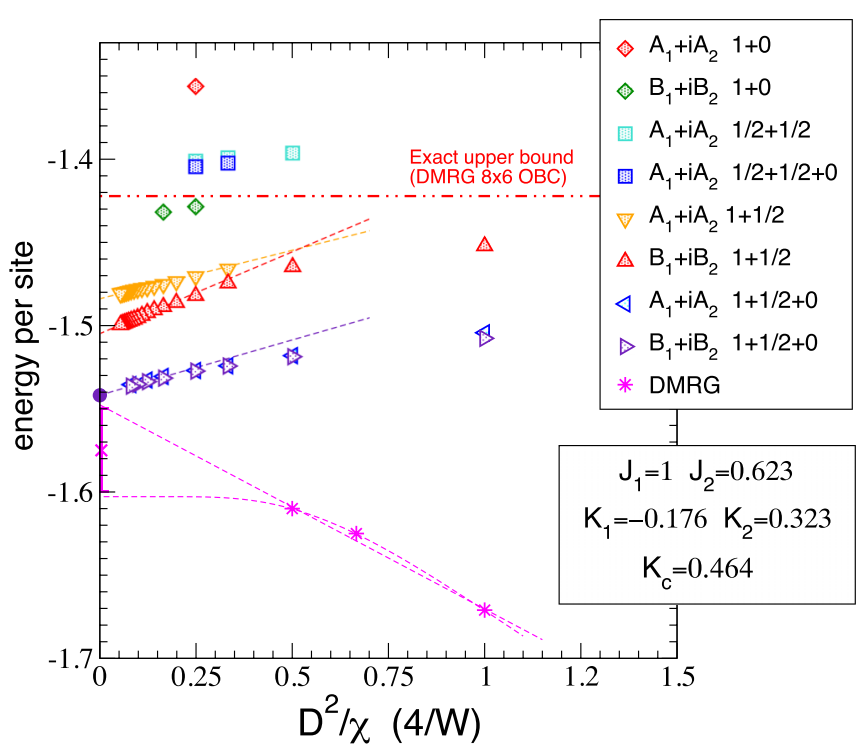

FIG. 3. iPEPS variational energies per site vs $D^{2} / \chi$. Results for $D=5$ and $D=6$ have been extrapolated to the $\chi \rightarrow \infty$ limit. A comparison to DMRG data obtained on cylinders of width $W$ (see text) and plotted vs $1 / W(\times 4)$ is shown. The DMRG data are extrapolated using linear and exponential fits, providing approximate bounds for the thermodynamic energy.

of the onset of topological GS degeneracy-is seen. Still, the three lowest singlets are indeed found at the $\Gamma$ point. We believe the cluster sizes are too small compared to some relevant bulk correlation length.

\section{B. Energy extrapolations}

Let us first consider the results for the iPEPS energy density plotted in Fig. 3 as a function of $D^{2} / \chi$, for $D=3,4,5$ and various classes corresponding to different virtual spaces. We only restrict here to the classes providing the best energies for a given choice of $D$. Note that the variational energy is optimized up to a maximal value of $\chi=\chi_{\text {opt }}$ which depends on $D$ (typically, $\chi_{\mathrm{opt}}=4 D^{2}=100$ for $D=5$ and $\chi_{\mathrm{opt}}=$ $3 D^{2}=108$ for $D=6$ ) and then a "frozen Ansatz" is used for $\chi>\chi_{\text {opt }}$. We observe that the energy density systematically decreases for increasing $\chi$ so that all data points can be considered as exact variational upper bounds. In fact, the data show a well-behaved scaling, almost perfectly linear in $1 / \chi$, so that accurate $\chi \rightarrow \infty$ extrapolations of the energy density can be obtained for each class. The best energies have been obtained for virtual spaces $1 \oplus \frac{1}{2}(D=5)$ and $1 \oplus \frac{1}{2} \oplus 0$ $(D=6)$. Note also that $1 \oplus 0(D=4)$ gives a better energy than $\frac{1}{2} \oplus \frac{1}{2} \otimes 0(D=5)$ so we believe the presence of a spin-1 in the virtual space is essential to get good variational Ansätze. Note also that the energy difference between the $\mathcal{A}$ and $\mathcal{B}$ PEPS decreases rapidly with increasing $D$, and become negligible for $D=6$. We believe the two Ansätze would describe exactly the same state in the $D \rightarrow \infty$ limit.

We then compare the PEPS energies to the DMRG results in Fig. 3. The DMRG energy densities computed on cylinders of various widths $W$ (see details above) have been (tentatively) extrapolated in the $W \rightarrow \infty$ limit showing good agreement with the $D=6$ iPEPS $\chi \rightarrow \infty$ extrapolation.

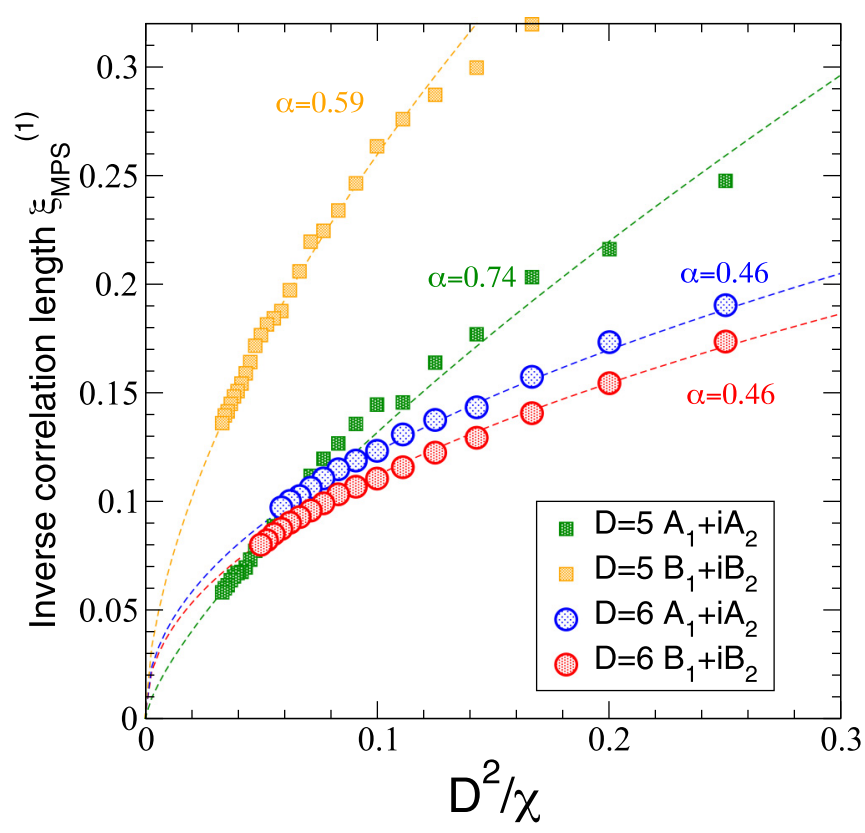

FIG. 4. Inverse of iPEPS (maximal) correlation length $1 / \xi_{\text {MPS }}^{(1)}$ (see text) vs $D^{2} / \chi$. The data for the four best optimized PEPS of bond dimension $D=5$ and 6 are shown with different symbols according to legend. Dashed lines are simple power-law fits, $1 / \xi \simeq$ $\left(D^{2} / \chi\right)^{\alpha}, \alpha<1$.

This indicates that our symmetric chiral PEPS provide good approximations for the true ground state in the thermodynamic limit, albeit with relatively small bond dimension $D$.

\section{Correlation lengths \\ 1. From the TM spectrum}

Correlation lengths can be obtained from the leading eigenvalues of the $\chi^{2} \times \chi^{2}$ transfer matrix $\mathcal{T}_{\text {MPS }}$ depicted in Fig. 1(f). Ordering the (real) eigenvalues as $t_{0}>\left|t_{1}\right|>\left|t_{2}\right|>\cdots$, one obtains decreasing correlation lengths $\xi_{\text {MPS }}^{(\mathrm{n})}, n \in \mathbb{N}^{+}$, defined as

$$
\xi_{\mathrm{MPS}}^{(\mathrm{n})}=1 / \ln \left(t_{0} /\left|t_{n}\right|\right) .
$$

Note that the leading eigenvalue is nondegenerate (and can be normalized to 1 ), while the subleading eigenvalues may be degenerate. This degeneracy provides information on the type of local operators the correlation length is associated to (see later). Alternatively, correlation lengths $\xi_{\mathrm{E}}^{(n)}$ can obtained in the same way from the $\chi^{2} D^{2} \times D^{2} \chi^{2}$ transfer matrix $\mathcal{T}_{\mathrm{E}}$, where an $E$ tensor is inserted between the two $T$ tensors [see Fig. 1(g)]. Since $T$ describes the exact environment fixed point in the limit $\chi \rightarrow \infty$, we expect $\xi_{\mathrm{E}}^{(n)} \rightarrow \xi_{\mathrm{MPS}}^{(\mathrm{n})}$ in that limit. Down to $\chi=D^{2}$, the two sets of (leading) correlation lengths remain quite similar so, in the following, we shall focus on $\xi_{\text {MPS }}^{(n)}$ for clarity.

The maximal correlation length $\xi_{\text {MPS }}^{(1)}$ has been computed in the best $D=5$ and 6 PEPS (optimized up to $\chi=3 D^{2}$ and $\chi=4 D^{2}$ ) for increasing CTMRG dimension $\chi=k D^{2}, k \in$ $\mathbb{N}^{+}$, up to $\chi=30 D^{2}$ and $\chi=17 D^{2}$, respectively. Results for $1 / \xi_{\text {MPS }}^{(1)}$ versus $D^{2} / \chi$ are shown in Fig. 4. Power-laws fit 


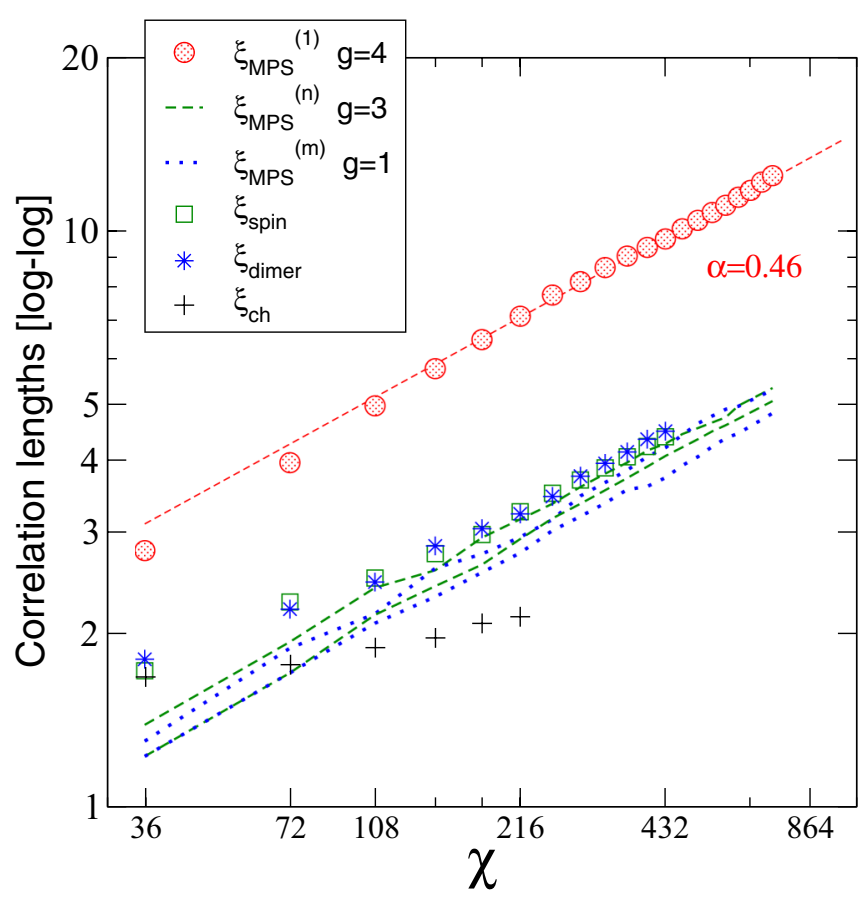

FIG. 5. Maximal and next four subleading correlation lengths $\xi_{\text {MPS }}^{(n)}, n=1, \ldots, 5$, in the $D=6 B_{1}+i B_{2}$ PEPS (optimized up to $\chi=108)$ vs $\chi$ in log-log scale, and sorted according to the degeneracy $g$ of the corresponding TM eigenvalue $\left|t_{n}\right|$. The dashed (straight) line corresponds to a simple power-law divergence, $\xi=A \chi^{\alpha}$ and $\alpha=0.46$. For comparison, spin and dimer correlation lengths are also shown.

the data well, suggesting that the maximal correlation length diverges as $\xi_{\text {MPS }}^{(1)} \propto \chi^{\alpha}$ when $\chi \rightarrow \infty$, with an exponent $\alpha<1$ (slow divergence). Hence, surprisingly, the bulk seems critical, unlike the FQHS analog. This is reminiscent of the spin-1/2 PEPS chiral spin liquid, which also seems to be critical (see comparison in Appendix A).

To get more insights on the nature of the correlations in the PEPS chiral SL, we have investigated the subleading correlation lengths $\xi_{\text {MPS }}^{(n)}, n>1$. Since the $A_{1}+i A_{2}$ and $B_{1}+$ $i B_{2} D=6$ chiral PEPS have very similar properties, we shall focus, from now on, on the $B_{1}+i B_{2} D=6$ PEPS. Results for the largest five correlation lengths are plotted in Fig. 5 on a log-log scale, showing a rather linear behavior over almost a decade. This confirms the (slow) power law increase $\xi \propto \chi^{\alpha}$, $\alpha \simeq 0.46$, also for the subleading correlation lengths.

\section{From the real-space correlation functions}

In order to identify the type of physical operators, these correlation lengths may be associated to, we have computed the spin-spin, (longitudinal) dimer-dimer, and chiral-chiral correlations versus distance (see Appendix B for details) and extracted the corresponding correlation lengths $\xi_{\mathrm{s}}, \xi_{\mathrm{d}}$, and $\xi_{\mathrm{ch}}$ from the long-distance behavior as illustrated in Fig. 6. We find that $\xi_{\mathrm{s}}$ and $\xi_{\mathrm{d}}$ are very close to the largest $\xi_{\text {MPS }}$ with degeneracy $g=3$ and $g=1$, respectively, consistent with triplet spin and singlet dimer operators. In contrast to $\xi_{\mathrm{s}}$ and $\xi_{\mathrm{d}}$, the chiral correlation length grows very slowly suggesting that the chiral correlation remains short-range. Interestingly,

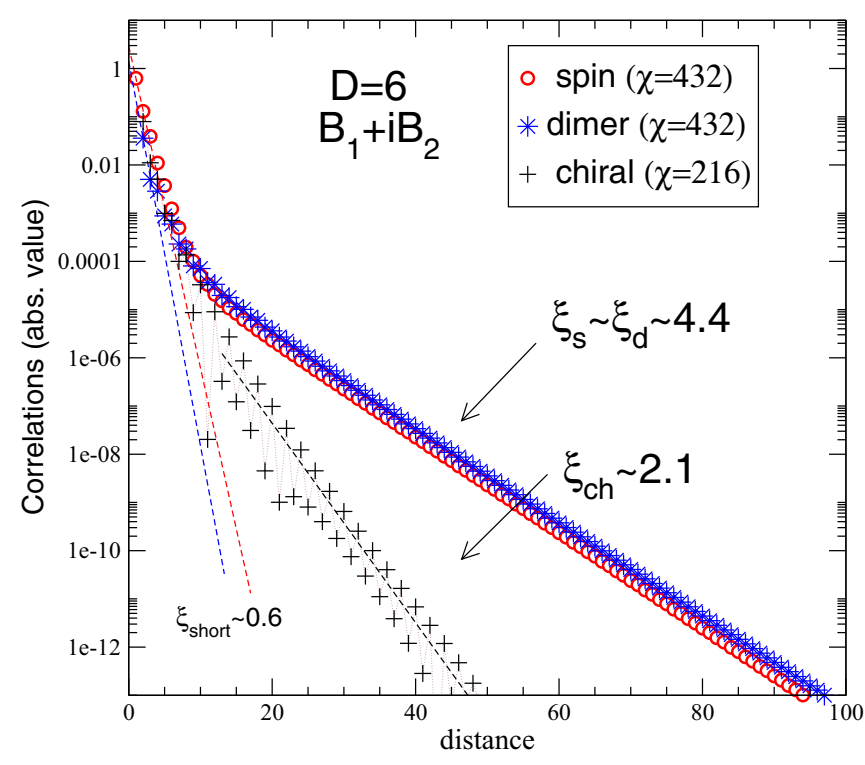

FIG. 6. Spin-spin, dimer-dimer, and chiral-chiral correlations (in absolute value) vs distance in the $D=6, B_{1}+i B_{2}$, chiral PEPS. Dashed lines are simple exponential fits of the short-range decay of the spin-spin and dimer-dimer correlations. The correlation lengths are extracted from the exponential decay at large distances.

the maximal correlation length $\xi_{\text {MPS }}^{(1)}$ is of degeneracy $g=4$ (which would naively correspond to a spin-3/2 operator) and hence cannot trivially be associated to a simple local operator acting on a group of physical sites but, perhaps, to chiral modes (see Sec. IV C) counterpropagating along the two chains of $T$ tensors of the long $\left(\mathcal{T}_{\text {MPS }}\right)^{\otimes N_{h}}$ ladder, $N_{h} \gg 1$.

Let us now examine in more details the form and the magnitude of the spin-spin and dimer-dimer correlation functions at all length scales. First, at short distance, we observe a rapid exponential fall-off characteristic of the lattice Moore-Read spin liquid (as for the spin-1/2 chiral PEPS, an Ansatz for the lattice KL state). The length scale associated to this shortrange behavior turned out to be very short, around $\xi_{\text {short }} \sim 0.6$, as seen in Fig. 6. More generally, one expects a sum of exponential contributions with a distribution of length scales. In other words, the spin-spin (or dimer-dimer) correlation function versus distance can be written as a discrete sum,

$$
C(d)=\sum_{\xi_{\text {short } \leqslant \xi \leqslant \xi_{s}}} w(\xi) \exp (-d / \xi),
$$

where the short-distance decay is characterized by $w\left(\xi_{\text {short }}\right) \simeq 1$ while, at long distance, the slower decay $\exp \left(-d / \xi_{\mathrm{s}}\right)$ takes over. Typically, we find that $\xi_{\mathrm{s}} \gg \xi_{\text {short }}$ and $w\left(\xi_{\mathrm{s}}\right) \ll 1$. In the limit $\chi \rightarrow \infty$, one expects that the spectrum of the transfer matrix becomes dense, so that one can use a continuous integral over all eigenvalues for computing $C(d)$, namely $C(d)=\int_{\xi} d \xi n(\xi) w(\xi) \exp \left(-\frac{d}{\xi}\right)$, where $n(\xi)$ is the density of state. Figure 5 suggests that the density of eigenvalues is constant in logarithmic scale so that $n(\xi) d \xi \sim \frac{d \xi}{\xi}$. In order to extract the possible functional form of the correlation function, it is now necessary to get the behavior of the weight function $w(\xi)$. To do so, we have plotted $w\left(\xi_{\mathrm{s}}\right)$ versus $\xi_{\mathrm{s}}$ and $w\left(\xi_{\mathrm{d}}\right)$ versus $\xi_{\mathrm{d}}$ in Figs. 7(a) and 7(b), using semi-log and 

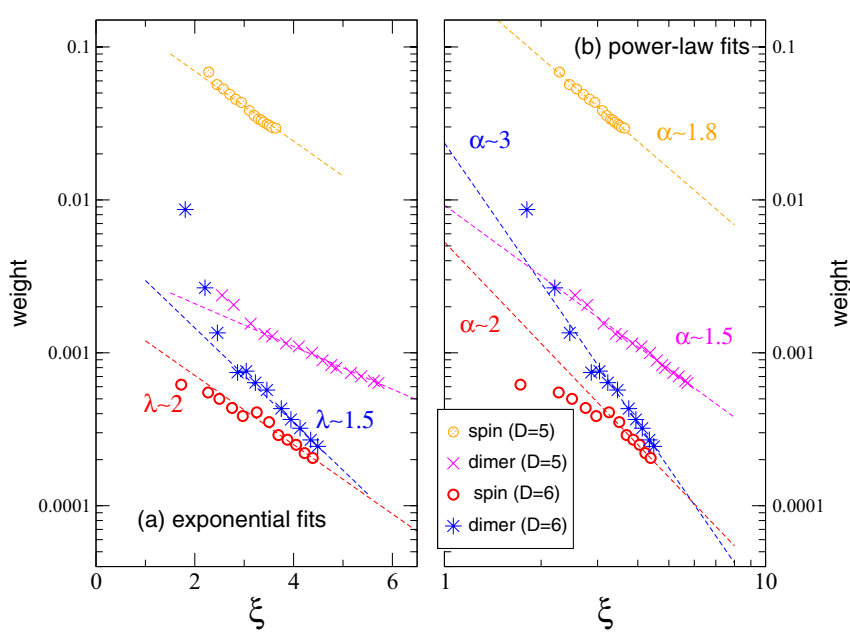

FIG. 7. Weight associated to the exponential decay with the maximal correlation length, as a function of the (maximal) correlation length, in the $B_{1}+i B_{2}$ chiral PEPS of bond dimension $D=5$ and 6. (a) Semilogarithmic plot. The straight lines are exponential fits $w(\xi) \sim \exp (-\xi / \lambda)$. (b) Log-log plot. The straight lines are powerlaw fits $w(\xi) \sim 1 / \xi^{\alpha}$.

log-log scales. In fact, because of the limited range of available maximal correlation lengths (obtained by varying the environment dimension $\chi$ ), both exponential and power-law fits give reasonable results, hence providing different answers for the long-range correlations. Let us examine each case separately.

Exponential decay of $w(\xi)$. Let us assume $w(\xi) \simeq$ $W_{D} \exp \left(-\xi / \lambda_{D}\right)$ in Eq. (6) as a legitimate Ansatz, where $\lambda_{D}$ is a new emerging length scale and $W_{D}$ is some amplitude, which both depend on the PEPS bond dimension $D$. Typically, our fits in Fig. 7 (a) give $\lambda_{6} \sim 2\left(\lambda_{6} \sim 1.5\right)$ for the spin-spin (dimer-dimer) correlations of the $D=6$ chiral PEPS. If this functional form is correct then $C(d) \sim$ $W_{D} \int_{\xi} \frac{d \xi}{\xi} \exp \left(-\frac{d}{\xi}-\frac{\xi}{\lambda_{D}}\right)$ will show a typical stretched exponential form at long distance,

$$
C(d) \sim W_{D} \exp \left[-2\left(d / \lambda_{D}\right)^{\frac{1}{2}}\right],
$$

up to a possible power-law prefactor in $d / \lambda_{D}$. Hence, in this case, the spin-spin and dimer-dimer correlations would decay faster than any power law. Interestingly, the same functional form was also proposed for the spin-1/2 chiral PEPS [51].

Power-law decay of $w(\xi)$. Let us now assume $w(\xi) \simeq$ $W_{D} / \xi^{\alpha}$ and substitute it in Eq. (6). In that case, a simple estimate of $C(d) \sim W_{D} \int_{\xi} \frac{d \xi}{\xi} \exp \left(-\frac{d}{\xi}\right) \frac{1}{\xi^{\alpha}}$ gives a power-law decay at long-distance of the form

$$
C(d) \sim W_{D}(1 / d)^{\alpha} .
$$

From the fits in Fig. 7(b) one gets estimates of the exponents of the spin-spin and dimer-dimer correlation functions, $\alpha_{s} \sim 2$ and $\alpha_{d} \sim 3$, respectively, for $D=6$.

Note that, in deriving (7) and (8), we have omitted potential oscillatory $(-1)^{d}$ behavior of some of the exponential terms of (6), which may reduce the range of the correlations. In any case, it is clear that the long-range tail of the spin-spin and dimer-dimer correlation functions, for a fixed bond dimension $D$, is of quite small magnitude proportional to $W_{D}$. Moreover,

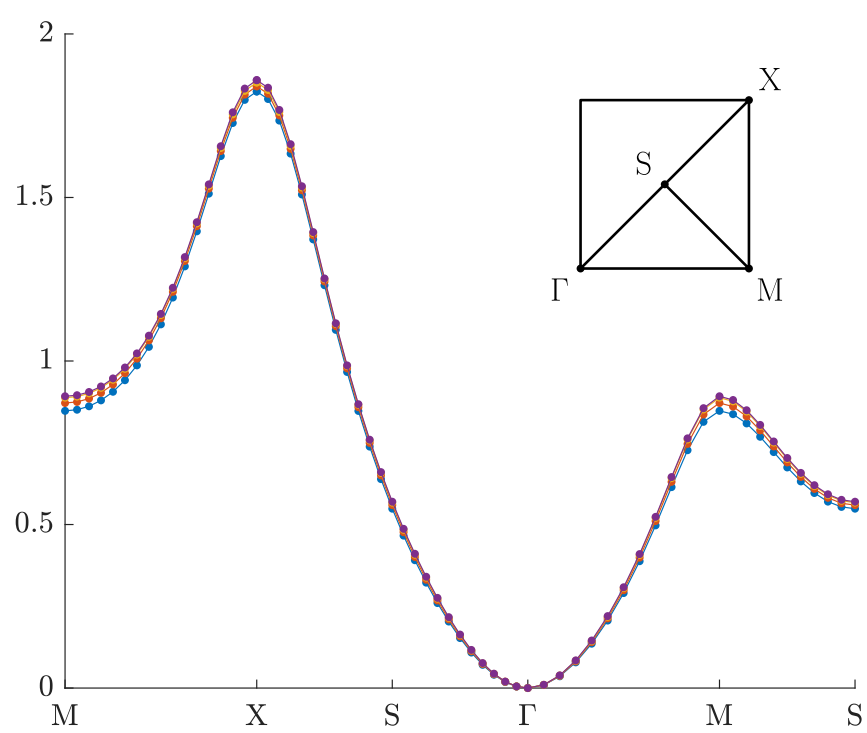

FIG. 8. Structure factor of the $D=6 B_{1}+i B_{2}$ chiral PEPS along the path in the Brillouin zone shown in the inset, for different values of the channel environment bond dimension $\chi=87$ (blue), 131 (red), 158 (yellow), and 185 (purple). Due to finite- $\chi$ effects we find slightly negative values (of the order of the truncation error) at the $\Gamma$ point; in order to better compare the different $\chi$ we have shifted the curves by this small offset.

from Figs. 7(a) and 7(b), it is also clear that the magnitude $W_{D}$ of $w(\xi)$ decreases strongly by increasing $D$. For example, $W_{D}$ associated to the spin correlation length gets smaller by two orders of magnitude, just by increasing $D$ from 5 to 6 . Hence we conjecture that this "gossamer" long-range tail is a finite- $D$ artifact of the chiral PEPS which should gradually disappear when increasing the bond dimension $D$.

\section{Spin structure factor}

The previous calculations of correlation lengths suggest the $D=6$ chiral PEPS exhibits a form of long-range tail, i.e., with a slower decay than a pure exponential function. In the case of a power-law decay of the spin-spin correlations like $C_{\mathrm{s}}(\mathbf{r})=W_{s}(1 /|\mathbf{r}|)^{\alpha} \exp \left(i \mathbf{Q}_{\mathrm{AF}} \cdot \mathbf{r}\right)$ [the antiferromagnetic wave vector $\mathbf{Q}_{\mathrm{AF}}=(\pi, \pi)$ is consistent with the data], the static spin structure factor $S(\mathbf{q})=\sum_{i} \exp \left(i \mathbf{q} \cdot \mathbf{r}_{\mathbf{i}}\right) C_{S}\left(\mathbf{r}_{\mathbf{i}}\right)$ would diverge when $\mathbf{q} \rightarrow \mathbf{Q}_{\mathrm{AF}}$, only if $\alpha<2$. In contrast, in the case of a stretched exponential form, as suggested by the data, no divergence is expected at any momentum.

In order to get more insight, we have computed the spin structure factor in the $D=6$ chiral PEPS, and results are shown in Fig. 8. The results do not show any sign of a divergence at any momentum, suggesting that the decay of the spin-spin correlations at long distance is relatively rapid and corresponds to a "gossamer" tail, consistent either with (7) or with (8), provided $\alpha \geqslant 2$.

\section{EDGE MODES}

\section{A. Expected $\mathrm{SU}(2)_{2}$ counting}

As suggested by Li and Haldane [63], the entanglement spectrum (ES) offers a very powerful tool to identify topological order in Abelian and non-Abelian liquid states since the 
TABLE II. Towers of states of the SU(2) $)_{2}$ WZW model, in each of the three sectors characterized by the primary fields $j=0, \frac{1}{2}, 1$ (listed in each column) and conformal weights $\frac{1}{4} j(j+1)$. Each line corresponds to a Virasoro level indexed by $n$. For each sector and each level, the (quasi-)degenerate states can be grouped in terms of exact $\mathrm{SU}(2)$ multiplets like $n_{0}(0)+n_{1}(1)+\cdots$ (meaning $n_{0}$ singlets, $n_{1}$ triplets, etc.).

\begin{tabular}{lccc}
\hline \hline$n \backslash j$ & 0 & $\frac{1}{2}$ & 1 \\
\hline 0 & $(0)$ & $\left(\frac{1}{2}\right)$ & $(1)$ \\
1 & $(1)$ & $\left(\frac{1}{2}\right)+\left(\frac{3}{2}\right)$ & $(0)+(1)$ \\
2 & $(0)+(1)+(2)$ & $2\left(\frac{1}{2}\right)+2\left(\frac{3}{2}\right)$ & $(0)+2(1)+(2)$ \\
3 & $(0)+3(1)+(2)$ & $4\left(\frac{1}{2}\right)+3\left(\frac{3}{2}\right)+\left(\frac{5}{2}\right)$ & $2(0)+3(1)+2(2)$ \\
4 & $3(0)+4(1)+3(2)$ & $6\left(\frac{1}{2}\right)+6\left(\frac{3}{2}\right)+2\left(\frac{5}{2}\right)$ & - \\
5 & $3(0)+8(1)+4(2)+(3)$ & $10\left(\frac{1}{2}\right)+10\left(\frac{3}{2}\right)+4\left(\frac{5}{2}\right)$ & - \\
\hline \hline
\end{tabular}

latter is in one-to-one correspondence with the edge states. Since our PEPS is expected to provide a reliable lattice representation of the Moore-Read FQH state (apart from-we believe-a spurious long-range "gossamer" tail in some bulk correlations), the edge modes should be described by a SU(2) WZW theory for which the ES will provide indisputable finger prints. The $\mathrm{SU}(2)_{2}$ WZW theory harbors three sectors whose towers of states - obtained by combining a bosonic (harmonic oscillator) mode with an independent Majoranafermion mode—are listed in Table II.

\section{B. Bulk-edge correspondence}

Let us now consider the chiral PEPS $\left|\Psi_{\text {PEPS }}\right\rangle$ on an infinitely long (horizontal) cylinder of finite circumference $N_{v}$, and a bipartition along a vertical cut into two right $(\mathrm{R})$ and left (L) semi-infinite cylinders. One can then define the reduced density matrix (RDM) $\rho$ of, let say, the $\mathrm{L}$ part by taking the trace of $\left|\Psi_{\text {PEPS }}\right\rangle\left\langle\Psi_{\text {PEPS }}\right|$ over the (physical) degrees of freedom of the R part. Rewriting the positive operator $\rho$ as $\exp \left(-H_{b}\right)$, where $H_{b}=-\ln \rho$ can be viewed as a "boundary" Hamiltonian, Li and Haldane conjectured [63] that the spectrum of $H_{b}$-dubbed entanglement spectrum-is in oneto-one correspondence with the actual edge spectrum of the partitioned system. Therefore the ES should exhibit crucial information on the nature of the chiral edge modes which, in turn, can provide a precise characterization of our chiral SL.

To compute the ES on a cylinder, one can use the PEPS bulk-edge correspondence theorem [64] that provides an exact relation between the RDM-whose support is the 2D halfcylinder physical Hilbert space—and a 1D (positive) operator $\sigma_{b}^{2}$ only acting on the $D^{N_{v}}$ virtual degrees of freedom of the "cut." The above fundamental relation involves an isometry that preserves the spectrum and [64]

$$
\sigma_{b}^{2}=\sqrt{\sigma_{L}^{T}} \sigma_{R} \sqrt{\sigma_{L}^{T}}
$$

where $\sigma_{L}\left(\sigma_{R}\right)$ is obtained from the cylinder TM [shown in Fig. 9(a)] left (right) leading eigenvector of dimension $\left(D^{2}\right)^{N_{v}}$, reshaped as a $D^{N_{v}} \times D^{N_{v}}$ matrix. Here, $\sigma_{L}^{T}=\sigma_{R}$, so that $\sigma_{b}=\sigma_{R}$. In previous work, on spin-1/2 chiral spin liquids [42], $\sigma_{b}^{2}$ was obtained for a simple chiral $D=3$ PEPS by exact tensor contractions, multiplying recursively the

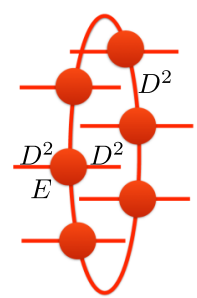

(a)

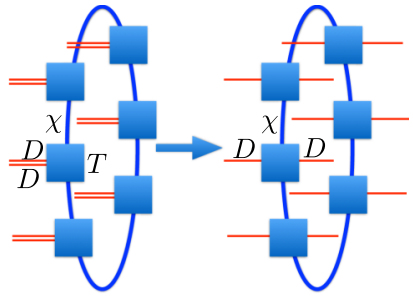

(b)
FIG. 9. (a) Cylinder transfer matrix. (b) Boundary (right) vector obtained by contracting $N_{v}$ (=6 here) environment $T$ tensors on a ring is reshaped into the $\operatorname{RDM} \rho_{R}$.

cylinder TM to get the leading eigenvector. For our $D=6$ chiral PEPS, such a procedure is no longer possible and one has to rely on approximate contraction schemes. Below, we describe two methods to compute $\sigma_{b}$, either using our previous knowledge of the iPEPS environment [65] or using a uniform MPS implementation.

\section{Finite $N_{v}$ calculation using CTMRG}

First, we construct the half-cylinder leading eigenvector using the previously obtained $T$ tensor, (i) by contracting $N_{v}$ such tensors on a ring of $N_{v}$ sites, and (ii) by reshaping this vector into a $\sigma_{b}$ matrix, as shown in Fig. 9(b). In this procedure, the CTMRG parameter $\chi$ becomes the only control parameter of the approximate calculation of the ES for finite $N_{v}$. Note, however, that, strictly speaking, the calculation does not become exact in the $\chi \rightarrow \infty$ limit since $T$ is computed for an infinite system. However, this procedure may be more advantageous in the sense that it may reduce some of the finite-size effects in the ES, in comparison to the exact contraction method.

In order to compute the ES, we first block-diagonalize the edge operator $\sigma_{b}$ using the exact symmetries of the PEPS, namely (i) its $\mathbb{Z}_{2}$ gauge symmetry-leading to even and odd sectors (ii) its full SU(2)-spin rotational invariance-leading to sectors labeled by the total spin projection $S_{z}$ and (iii) its translational invariance-leading to sub-sectors labeled by the edge momentum $K=n \frac{2 \pi}{N_{v}}, n \in \mathbb{Z}$. First, as for the spin- $1 / 2$ chiral PEPS [42,43], the even (odd) topological gauge sector only contains the integer (half-integer) $S_{z}$ sectors. Secondly, because of the chosen $\pi$-spin rotation on the sites of the B-sublattice (in order to deal with the same unique tensor on the A and B sites), the $S_{z}$ operator acquires a minus sign under an odd number of lattice translations, so that only (i) $\left|S_{z}\right|$ and $K$ or (ii) $S_{z}$ and $K[\bmod \pi]$ can be considered simultaneously.

The entanglement spectrum of the $D=6 B_{1}+i B_{2}$ PEPS has been computed on a $N_{v}=6$ site ring from the $T$ environment tensor with $\chi=D^{2}=36, \chi=4 D^{2}=144$, and $\chi=6 D^{2}=216$. Data for $\chi=216$ are shown in Fig. 10 in the range $[-\pi, \pi)$ (the complete spectrum for $\chi=144$ is also shown in Fig. 15 in the Appendix C). Two low (quasi-)energy chiral branches emerge, linearly dispersing in one direction only, separated by momentum $\Delta K=\pi$. By grouping together the degenerate energy levels at $K$ and $K+\pi$, one obtains exact $\mathrm{SU}(2)$ multiplets that can be labeled by their 


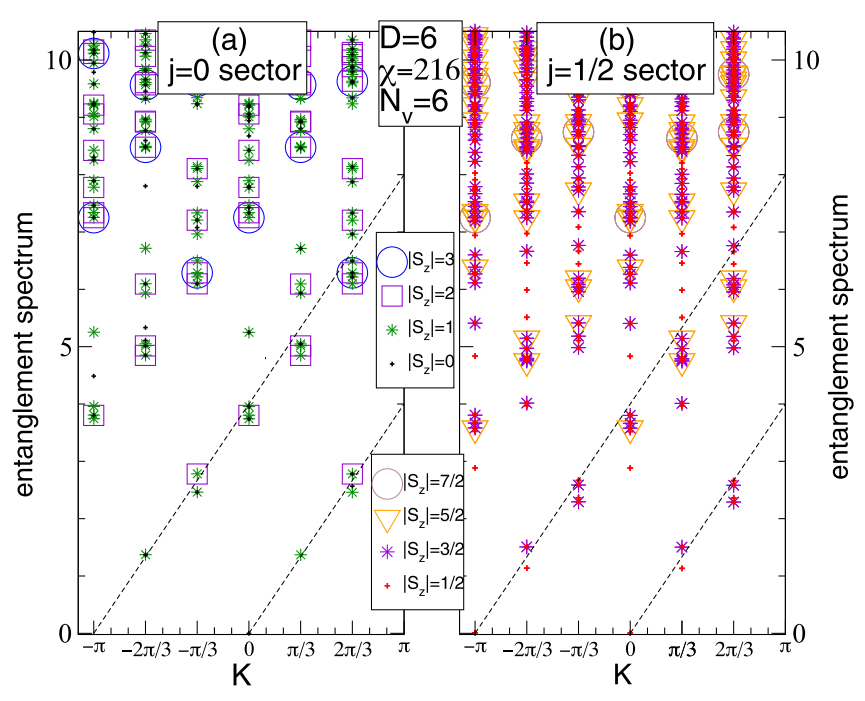

FIG. 10. Entanglement spectrum of the $B_{1}+i B_{2} D=6$ chiral PEPS for $N_{v}=6$ (see text) vs edge momentum $K$, computed for $\chi=216$. Different symbols correspond to different values of $\left|S_{z}\right|$, showing that the spectrum is composed of exact SU(2) multiplets with integer (a) and half-integer spins (b). Dashed lines correspond to the low-energy chiral CFT modes.

total spin $S$ quantum number. ${ }^{2}$ The same spectrum can then be plotted for $K \in[0, \pi$ [, mod $\pi$, labeling now the levels according to their spin $S$, as shown in Fig. 11. In this way, in each topological sector, the two branches merge into a unique chiral branch composed of groups of quasidegenerate exact SU(2) multiplets, labeled as $\oplus_{S_{\min }}^{S_{\max }} n_{S}(S), n_{S} \in \mathbb{N}$. Examining carefully each group of multiplets, for increasing momentum $K=$ $n \frac{2 \pi}{N_{v}}$, we find that their content agrees exactly-at least up to the fourth level - the prediction of the $\mathrm{SU}(2)_{2} \mathrm{WZW}$ conformal field theory of central charge $c=3 / 2$, characterized by a bosonic mode combined with an Ising anyon (or "Majorana fermions"). A comparison with the ES of the spin- $1 / 2$ CSL is shown in Appendix C, showing a very distinct $\mathrm{SU}(2)_{1}$ CFT counting.

Note that the third $j=1$ topological tower cannot be derived straightforwardly since, it probably requires the insertion of a string of $\mathbb{Z}_{2}$ "vison" operators in the cylinder direction (see Ref. [42] for the case of the simple spin-1/2 CSL). The representation of the vison operator in the dimension- $\chi$ fixed-point basis is not known.

\section{Uniform MPS calculation}

We can also characterize the entanglement spectrum using uniform MPS techniques. Similarly as before (see Fig. 9), we take the fixed point (leading eigenvector) of the PEPS transfer matrix and interpret it as a matrix-product operator $\rho$ (with bond dimension $\chi$ ) representing the boundary Hamiltonian as $\rho=\exp \left(-H_{b}\right)$. Here we work in the thermodynamic limit

\footnotetext{
${ }^{2}$ Note that the ES eigenvalues in the odd sector of the half-integer spin multiplets are all exactly twofold degenerate as for the spin-1/2 chiral spin liquid $[42,43]$ due to an interplay between $S U(2)$ and space-group symmetries [70].
}
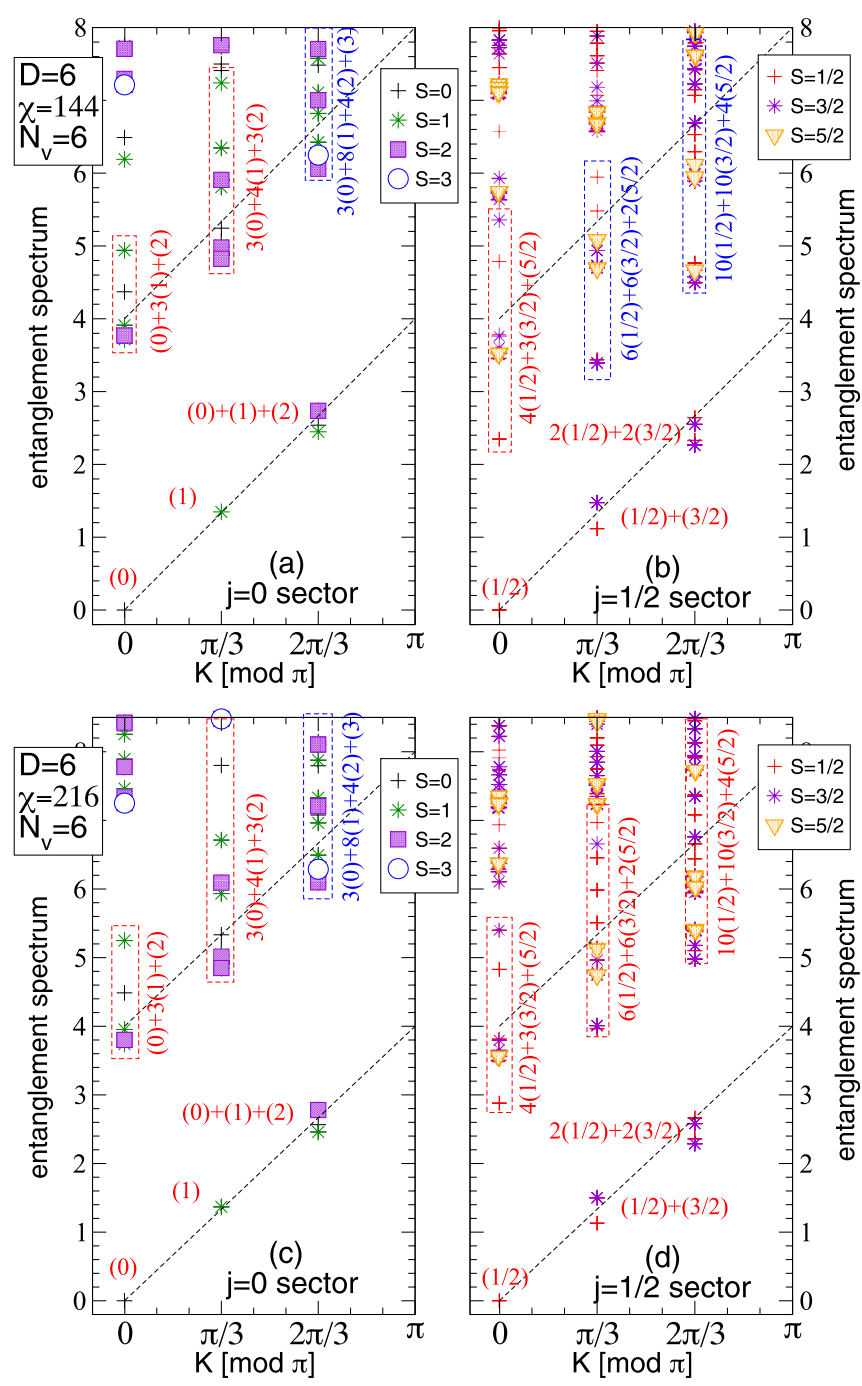

FIG. 11. Close-up of the low (quasi-)energy entanglement spectrum of the $D=6$ chiral PEPS for $N_{v}=6 \mathrm{vs} K[\bmod \pi]$ (see text). Comparison of the spectra for $\chi=144$ [(a) and (b)] and $\chi=216$ [(c) and (d)] is shown showing an almost convergence with $\chi$. The expected $j=0$ and $j=1 / 2$ chiral modes of the WZW SU(2) theory appear at the bottom of the $\mathbb{Z}_{2}$-even [(a) and (c)] and $\mathbb{Z}_{2}$-odd [(b) and (d)] sectors of the ES, respectively. The SU(2)-multiplet content of each group of levels is indicated, in agreement with the CFT prediction (except for the blue boxes where a few levels are missing).

directly, such that the leading eigenvector of this MPO (corresponding to the ground state of the boundary Hamiltonian $H_{b}$ ) can, again, be approximated as a uniform matrix product state. In particular, we can plot the scaling of the bipartite entanglement entropy of this uniform MPS as a function of its correlation length, which is known [66] to be related to the central charge as $S \propto \frac{c}{6} \ln (\xi)$. As shown in Fig. 12(a), this provides clear evidence that the boundary theory is described by a CFT with central charge $c=3 / 2$. In addition, we can compute the spectrum of $H_{b}$ by applying the quasiparticle excitation Ansatz [67,68]. In Fig. 12(b), we have plotted the entanglement spectrum, showing the signature of a chiral spectrum. The presence of a very steep "left-moving" branch 


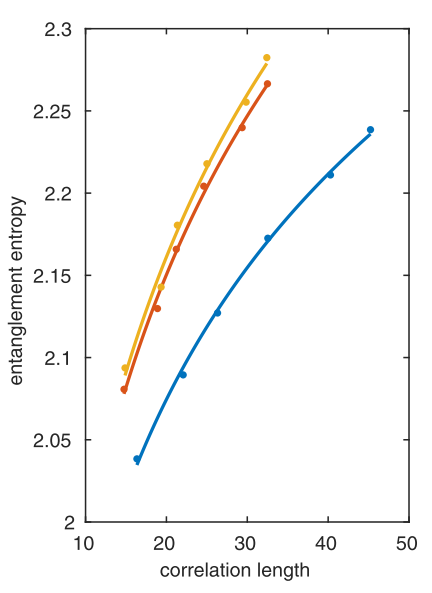

(a)

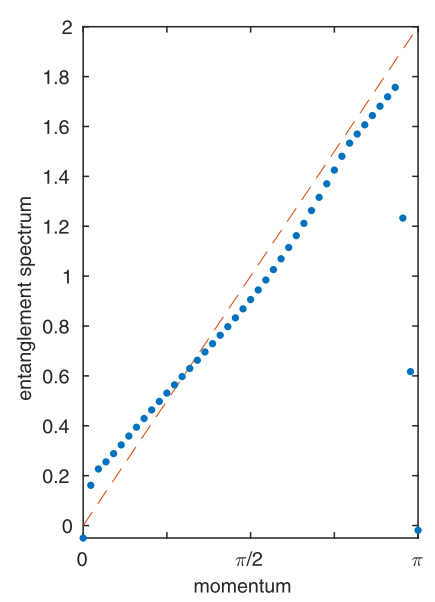

(b)
FIG. 12. (a) The bipartite entanglement entropy as a function of the correlation length for MPS approximations (with increasing bond dimension $\left.\chi^{\prime}=50 \rightarrow 100\right)$ of the fixed point of $\rho \approx \exp \left(-H_{b}\right)$; the three data sets are for different values of the bond dimension $\chi$ of the MPO approximation for $\rho: \chi=87$ (blue), 131 (red), and 158 (yellow). The lines are fits to the data points with $S \propto \frac{c}{6} \ln (\xi)$, where we find $c_{\chi=87}=1.187, c_{\chi=131}=1.429$, and $c_{\chi=158}=1.462$. (b) The spectrum of $H_{b}$ as computed with the quasiparticle Ansatz on the MPO approximation $\rho \approx \exp \left(-H_{b}\right)$ with bond dimension $\chi=158$ in the thermodynamic limit.

and a small modulation on top of the linear dispersion are the consequence of the finite- $\chi$ approximation of $\rho$.

\section{SUMMARY, DISCUSSION, AND OUTLOOK}

Our iPEPS study provides the first investigation of a (finetuned) spin-1 frustrated Heisenberg model on the square lattice, which includes a time-reversal breaking plaquette term. The ES and the scaling of the entanglement entropy provide smoking gun evidence of $\mathrm{SU}(2)_{2}$ chiral edge modes with central charge $c=3 / 2$, consistent with a bulk non-Abelian CSL realizing, on the lattice, the Moore-Read FQH state.

However, our results also point towards long-range behavior of some bulk correlations (such as spin-spin or dimerdimer correlation functions) which may be algebraic or more rapidly decaying (as, e.g., for a stretched exponential). We argue that, although this behavior might be generic in a chiral PEPS (see the exact proof for Gaussian PEPS in Ref. [52]), it is a spurious artifact which does not constitute a real obstruction for accurate investigation of truly gapped CSL ground states of simple (frustrated) quantum spin Hamiltonians. First, we note that chiral edge modes can be truly gapless only if the effective one-dimensional PEPS boundary Hamiltonian $H_{b}$ (acting on the virtual space) is long range, which in turn implies, from the PEPS bulk-edge correspondence [64], that the (maximal) bulk correlation length indeed diverges. However, our results also show that this "necessary" bulk criticality only manifests itself in the form of gossamer tails in some physical correlation functions, whose magnitude is already quite small for $D=6$ and even seems to decrease rapidly with the tensor bond dimension. This suggests that "gossamer" tails will gradually disappear when $D \rightarrow \infty$, with no practical effect to faithfully approximate a gapped CSL (as far as energy, short and intermediate range correlations, edge mode physics, etc., are concerned) with a finite- $D$ chiral PEPS. Therefore the PEPS formalism seems to be an unbiased efficient method to investigate other non-Abelian CSL in higher spin SU(2)-invariant HAFM (with or without explicit time-reversal breaking) and in SU(N) models.

Lastly, we comment on possible experimental realizations of Hamiltonian (1). Let us start from a two-orbital Hubbard model with on-site Hubbard and (ferromagnetic) Hund couplings, and hoppings on both NN and NNN sites, respectively. In some limit of very large Hubbard and Hund couplings, only localized spin-1 degrees of freedom can be retained on the sites and two-site spin interactions appear in second order in the hoppings. Moreover, if an orbital flux is included in the plaquettes of the square lattice (breaking time-reversal symmetry), then chiral terms appear in third order of the hoppings. As suggested in Refs. [32,33] (although for the spin-1/2 case), such Hamiltonians can be realized using, e.g., ultracold atoms loaded on optical lattices in the presence of a synthetic gauge field [69].

\section{ACKNOWLEDGMENTS}

This project is supported in part by the TNSTRONG ANR grant (French Research Council). This work was granted access to the HPC resources of CALMIP and GENCI supercomputing centers under the allocation 2017-P1231 and A0030500225, respectively. LV was supported by the Flemish Research Foundation. We acknowledges inspiring conversations with F. Becca, A. Läuchli, G. Misguich, P. Pujol, G. Sierra, S. Simon and A. Sterdyniak. We are also thankful to B. Estienne for help in establishing the content of the $\mathrm{SU}(2)_{2}$ CFT tower of states.

\section{APPENDIX A: COMPARISON OF THE CORRELATION LENGTHS IN THE SPIN-1 AND THE SPIN-1/2 CHIRAL HAFM}

The maximal correlation length $\xi_{\mathrm{E}}^{(1)}$ of the spin-1 chiral PEPS has been obtained from the two largest eigenvalues of the $\mathcal{T}_{\mathrm{E}}$ transfer matrix depicted in Fig. 1(g). Its divergence with $\chi$ was shown to track the one of the dimer-dimer correlation length. In Fig. 13, we compare $\xi_{\mathrm{E}}^{(1)}$ in the spin-1 $D=5$ and $D=6$ chiral PEPS to the dimer-dimer correlation length in the related spin-1/2 chiral HAFM, for two choices of the model parameter considered in Ref. [51]. The behaviors of the spin-1/2 and spin-1 chiral PEPS are very similar, both being consistent with a power-law divergence (as shown by the dashed line fits).

\section{APPENDIX B: COMPUTING CORRELATION FUNCTIONS WITH IPEPS}

Correlation between any two (local) operators can be obtained by using infinitely long strips (running, let say, along the $x$ direction) bounded on each side by lines of fixedpoint $T$ environment tensors, as shown in Figs. 14(a)-14(e). Depending on the type of operator to be considered, a single line or two lines of $E$ tensors have to be inserted between 

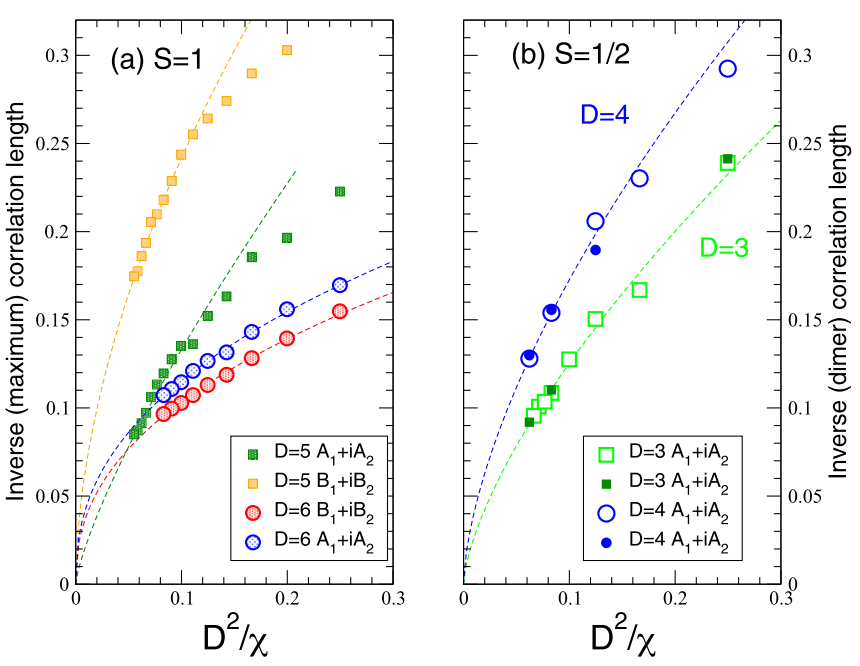

FIG. 13. Comparison between the inverse iPEPS (maximal) correlation length $\xi_{\mathrm{E}}^{(1)}$ (see text) of the spin-1 chiral HAFM (a) and the inverse iPEPS (dimer) correlation length $\xi_{\text {dimer }}$ of the spin-1/2 chiral HAFM (b). The data for the four best optimized spin-1 PEPS of bond dimension $D=5$ and 6 are shown in (a). In (b), open and closed symbols correspond to two different sets of the Hamiltonian parameters given by $J_{1}=K_{c}=1, J_{2}=0$ (open symbols) and $J_{1}=$ $0.89, J_{2}=0.42$, and $K_{c}=0.375$ (closed symbols). Dashed lines are simple power-law fits, $1 / \xi \simeq\left(D^{2} / \chi\right)^{\alpha}, \alpha<1$.

the two (infinitely long) boundaries. The new fixed point environment on the left side (right side) of the left-most (rightmost) operator is then constructed, as shown in Figs. 14(a) and 14(b). Two single-site, two-site, or four-site operators like

$$
\begin{aligned}
O_{i}^{\text {spin }}= & \mathbf{S}_{i} \\
O_{i}^{\text {dimer }}= & \mathbf{S}_{i} \cdot \mathbf{S}_{i+e_{x}} \\
O_{i}^{\text {chiral }}= & \mathbf{S}_{i} \cdot\left(\mathbf{S}_{i+e_{x}} \times \mathbf{S}_{i-e_{y}}\right)+\mathbf{S}_{i} \cdot\left(\mathbf{S}_{i+e_{x}-e_{y}} \times \mathbf{S}_{i-e_{y}}\right) \\
& +\mathbf{S}_{i} \cdot\left(\mathbf{S}_{i+e_{x}} \times \mathbf{S}_{i+e_{x}-e_{y}}\right) \\
& +\mathbf{S}_{i+e_{x}} \cdot\left(\mathbf{S}_{i+e_{x}-e_{y}} \times \mathbf{S}_{i-e_{y}}\right)
\end{aligned}
$$

where $e_{x}\left(e_{y}\right)$ is the unit vector along (perpendicular to) the strip are then inserted at a distance $d$, as shown in Figs. 14(c) and 14(e). The corresponding correlation functions can then be computed by applying $d-1$ or $d-2$ times the TM between the two operators. Note that, when the local operator has a finite expectation value, the connected correlation function is computed, i.e., making the replacements $O_{i}^{\text {dimer }} \rightarrow$ $O_{i}^{\text {dimer }}-\left\langle O_{i}^{\text {dimer }}\right\rangle$ and $O_{i}^{\text {chiral }} \rightarrow O_{i}^{\text {chiral }}-\left\langle O_{i}^{\text {chiral }}\right\rangle$.

\section{APPENDIX C: ADDITIONAL DATA ON THE ENTANGLEMENT SPECTRUM FOR $N_{v}=6$}

For completeness, we provide in Fig. 15 the full entanglement spectra in the $j=0$ and $j=1 / 2$ topological sectors, computed from a ring of $N_{v} T$ environment tensors at $\chi=$ 144. Note that in the gauge chosen to write down the PEPS (convenient for CTMRG), the generator of the spin-SU(2) group is invariant under even translation and $S_{z} \rightarrow-S_{z}$ under odd translations. As a consequence, $S_{z}$ is defined up to a sign. This also implies that the $2 S+1$ states of the $\mathrm{SU}(2)$ spin- $S$

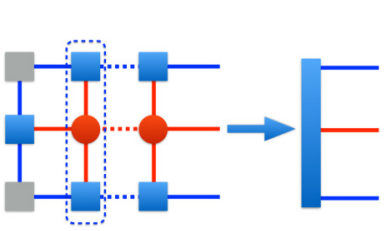

(a)

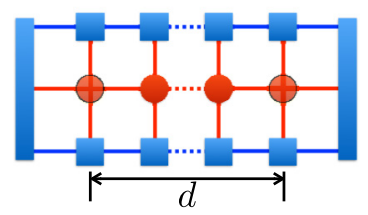

(c)

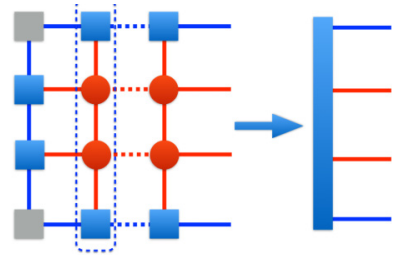

(b)

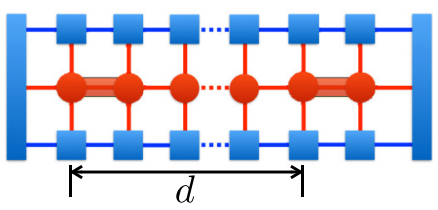

(d)

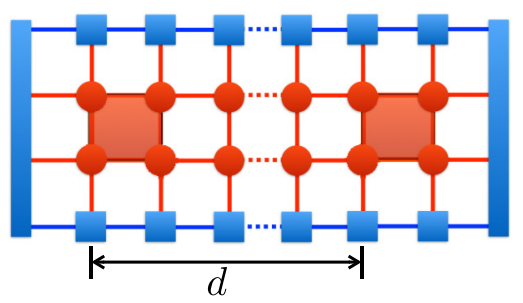

(e)

FIG. 14. The fixed point environments for strips with a single line (a) or with two lines (ladder) (b) of $E$ tensors are obtained as leading eigenvectors of the transfer matrices marked by dotted boxes. Strips to compute spin-spin (c), (longitudinal) dimer-dimer (d), and chiral-chiral (e) correlations, inserting two spin, dimer or chiral operators within the $E$ tensor(s) at distance $d$.

multiplets (labelled by $\left|S_{z}\right|$ instead of $S_{z}$ ) are split between momentum $k$ and momentum $k+\pi$, as can be checked directly in Fig. 15. Note that the two dashed lines at low energy

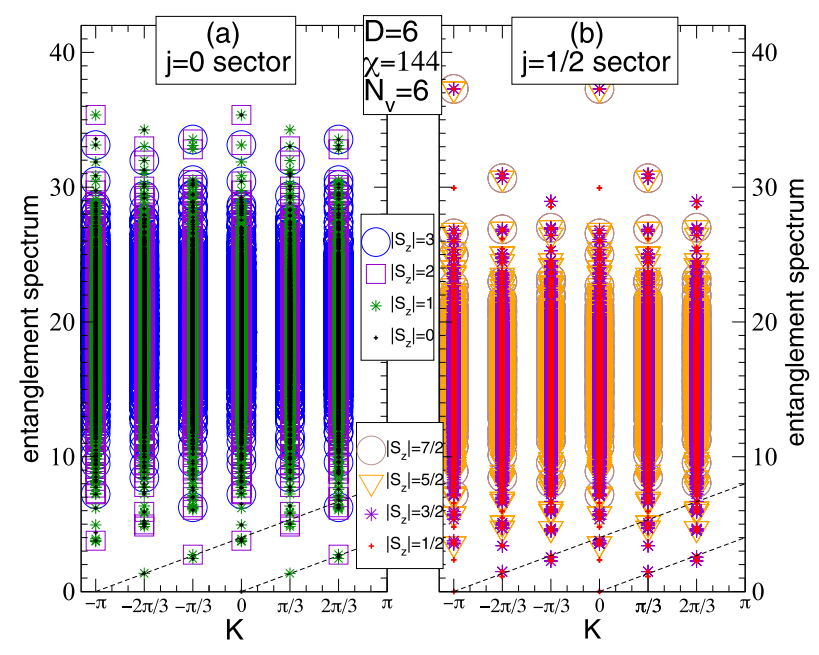

FIG. 15. Complete entanglement spectrum of the $B_{1}+i B_{2} D=$ 6 chiral PEPS for $N_{v}=6$ vs edge momentum $K$. Different symbols correspond to different values of $\left|S_{z}\right|$, showing that the spectrum is composed of exact SU(2) multiplets with integer (a) and half-integer spins (b). Dashed lines correspond to the low-energy chiral CFT modes. 

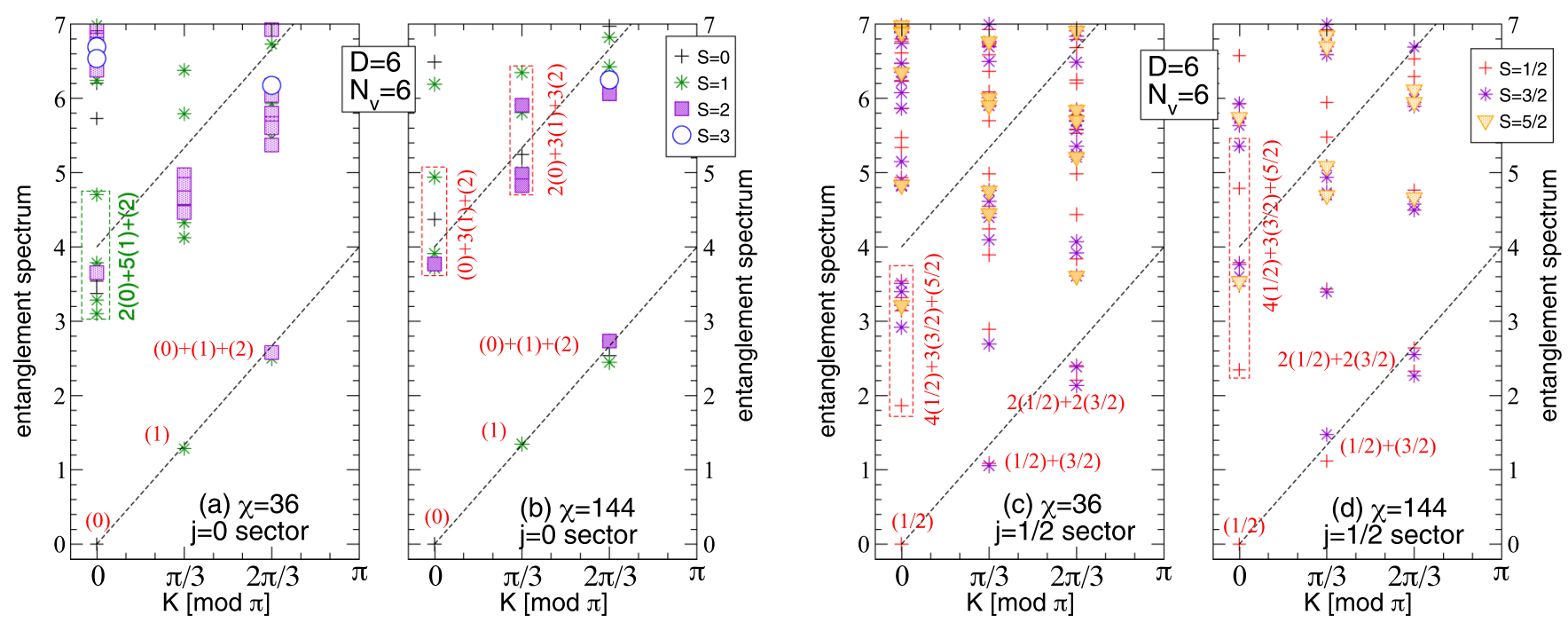

FIG. 16. Comparison between entanglement spectra of the $B_{1}+i B_{2} D=6$ chiral PEPS (for $N_{v}=6$ ) computed using the iPEPS $T$ tensor obtained either with $\chi=D^{2}=36\left((\mathrm{a})\right.$ and (c)) or with $\chi=4 D^{2}=144((\mathrm{~b})$ and (d)).

correspond, in fact, to a unique chiral mode as it becomes clear by plotting the spectrum in the reduced Brillouin zone $[0, \pi[$ (see main text). Note that the spectrum can be "unfolded" and plotted in the full Brillouin zone while still keeping the full SU(2) multiplet structure by using a different gauge for the PEPS that does not preserve the full rotation symmetry of the local tensor [70].

Since the ES of the $D=6$ chiral PEPS is computed using the CTMRG fixed-point tensor $T$, a systemic finite- $\chi$ error seems inerrant to the calculation. Nevertheless, one can argue that the results should saturate once, typically, the correlation length $\xi_{\mathrm{MPS}}^{(1)}(\chi)$ becomes bigger than the system size $N_{v}$. The ES obtained for $\chi=36$ and 144, for which $\xi_{\mathrm{MPS}}^{(1)} \sim 2.8$ and $\xi_{\text {MPS }}^{(1)} \sim 5.8$, respectively, are compared in Figs. 16(a)-16(d). Clearly, the spectra for $\chi=36$ are not fully converged, e.g., the fourth $j=0$ Virasoro level (shown by the green box) containing spurious $S=0$ and $S=1$ multiplets. The correct $\mathrm{SU}(2)_{2}$ counting is obtained for more levels for $\chi=144$. Since $\xi_{\mathrm{MPS}}^{(1)}(\chi=144) \sim N_{v}$, we expect that this spectrum is already quite close from the exact ES of the $D=6$ chiral PEPS on an infinitely-long cylinder of perimeter $N_{v}=6$.
[1] H. L. Stormer and D. C. Tsui, The quantized Hall effect, Science 220, 1241 (1983).

[2] X. G. Wen, Topological orders in rigid states, Int. J. Mod. Phys. B 04, 239 (1990).

[3] X.-G. Wen, Topological order: From long-range entangled quantum matter to a unified origin of light and electrons, ISRN Condens. Matter Phys. 2013, 198710 (2013).

[4] X. G. Wen, Gapless boundary excitations in the quantum Hall states and in the chiral spin states, Phys. Rev. B 43, 11025 (1991).

[5] X.-G. Wen, Theory of the edge states in fractional quantum hall effects, Int. J. Mod. Phys. B 06, 1711 (1992).

[6] B. I. Halperin, Statistics of Quasiparticles and the Hierarchy of Fractional Quantized Hall States, Phys. Rev. Lett. 52, 1583 (1984).

[7] R. B. Laughlin, Anomalous Quantum Hall Effect: An Incompressible Quantum Fluid with Fractionally Charged Excitations, Phys. Rev. Lett. 50, 1395 (1983).

[8] X. G. Wen, Non-Abelian Statistics in the Fractional Quantum Hall States, Phys. Rev. Lett. 66, 802 (1991).

[9] C. Nayak, S. H. Simon, A. Stern, M. Freedman, and S. Das Sarma, Non-abelian anyons and topological quantum computation, Rev. Mod. Phys. 80, 1083 (2008).
[10] G. Moore and N. Read, Nonabelions in the fractional quantum Hall effect, Nucl. Phys. B 360, 362 (1991).

[11] A. Yu Kitaev, Unpaired Majorana fermions in quantum wires, Phys. Usp. 44.10S, 131 (2001).

[12] J. Alicea, New directions in the pursuit of Majorana fermions in solid state systems, Rep. Prog. Phys. 75, 076501 (2012).

[13] C. Repellin, B. A. Bernevig, and N. Regnault, $\mathbb{Z}_{2}$ fractional topological insulators in two dimensions, Phys. Rev. B 90, 245401 (2014).

[14] J. Maciejko and G. A. Fiete, Fractionalized topological insulators, Nat. Phys. 11, 385 (2015).

[15] V. Kalmeyer and R. B. Laughlin, Equivalence of the Resonating-Valence-Bond and Fractional Quantum Hall States, Phys. Rev. Lett. 59, 2095 (1987).

[16] X. G. Wen, F. Wilczek, and A. Zee, Chiral spin states and superconductivity, Phys. Rev. B 39, 11413 (1989).

[17] B. Bauer, L. Cincio, B. P. Keller, M. Dolfi, G. Vidal, S. Trebst, and A. W. W. Ludwig, Chiral spin liquid and emergent anyons in a kagome lattice Mott insulator, Nat. Commun. 5, 5137 (2014).

[18] A. Wietek, A. Sterdyniak, and A. M. Läuchli, Nature of chiral spin liquids on the kagome lattice, Phys. Rev. B 92, 125122 (2015). 
[19] A. Wietek and A. M. Läuchli, Chiral spin liquid and quantum criticality in extended $s=\frac{1}{2}$ Heisenberg models on the triangular lattice, Phys. Rev. B 95, 035141 (2017).

[20] S.-S. Gong, W. Zhu, J.-X. Zhu, D. N. Sheng, and K. Yang, Global phase diagram and quantum spin liquids in a spin- $\frac{1}{2}$ triangular antiferromagnet, Phys. Rev. B 96, 075116 (2017).

[21] Y.-C. He, D. N. Sheng, and Y. Chen, Chiral Spin Liquid in a Frustrated Anisotropic Kagome Heisenberg Model, Phys. Rev. Lett. 112, 137202 (2014).

[22] A. Kitaev, Anyons in an exactly solved model and beyond, Ann. Phys. 321, 2 (2006).

[23] M. Greiter and R. Thomale, Non-Abelian Statistics in a Quantum Antiferromagnet, Phys. Rev. Lett. 102, 207203 (2009)

[24] Z.-X. Liu, H.-H. Tu, Y.-H. Wu, R.-Q. He, X.-J. Liu, Y. Zhou, and T.-K. $\mathrm{Ng}$, Non-abelian $s=1$ chiral spin liquid on the kagome lattice, Phys. Rev. B 97, 195158 (2018).

[25] P. Lecheminant and A. M. Tsvelik, Lattice spin models for nonabelian chiral spin liquids, Phys. Rev. B 95, 140406 (2017).

[26] D. F. Schroeter, E. Kapit, R. Thomale, and M. Greiter, Spin Hamiltonian for Which the Chiral Spin Liquid is the Exact Ground State, Phys. Rev. Lett. 99, 097202 (2007).

[27] R. Thomale, E. Kapit, D. F. Schroeter, and M. Greiter, Parent hamiltonian for the chiral spin liquid, Phys. Rev. B 80, 104406 (2009).

[28] A. E. B. Nielsen, J. I. Cirac, and G. Sierra, Laughlin SpinLiquid States on Lattices Obtained from Conformal Field Theory, Phys. Rev. Lett. 108, 257206 (2012).

[29] M. Greiter, D. F. Schroeter, and R. Thomale, Parent Hamiltonian for the non-Abelian chiral spin liquid, Phys. Rev. B 89, 165125 (2014).

[30] I. Glasser, J. I. Cirac, G. Sierra, and A. E. B. Nielsen, Exact parent Hamiltonians of bosonic and fermionic Moore-Read states on lattices and local models, New J. Phys. 17, 082001 (2015).

[31] B. Herwerth, G. Sierra, H.-H. Tu, J. I. Cirac, and A. E. B. Nielsen, Edge states for the Kalmeyer-Laughlin wave function, Phys. Rev. B 92, 245111 (2015).

[32] A. E.B. Nielsen, G. Sierra, and J. I. Cirac, Local models of fractional quantum Hall states in lattices and physical implementation, Nat. Commun. 4, 2864 (2013).

[33] A. E. B. Nielsen, G. Sierra, and J. I. Cirac, Optical-lattice implementation scheme of a bosonic topological model with fermionic atoms, Phys. Rev. A 90, 013606 (2014).

[34] Z. Zhu, I. Kimchi, D. N. Sheng, and L. Fu, Robust non-abelian spin liquid and a possible intermediate phase in the antiferromagnetic Kitaev model with magnetic field, Phys. Rev. B 97, 241110 (2018).

[35] J. I. Cirac and F. Verstraete, Renormalization and tensor product states in spin chains and lattices, J. Phys. A: Math. Theor. 42, 504004 (2009).

[36] J. I. Cirac, Entanglement in many-body quantum systems, in Many-Body Physics with Ultracold Atoms ( Les Houches school, 2010).

[37] R. Orús, A practical introduction to tensor networks: Matrix product states and projected entangled pair states, Ann. Phys. 349, 117 (2014).

[38] N. Schuch, Condensed matter applications of entanglement theory, in Quantum Information Processing: Lecture Notes, Schriften des Forschungszentrums Jülich. Reihe Schlüsseltech- nologien / Key Technologies, 44th IFF Spring School (David P. DiVincenzo, Forschungszentrum Jülich, 2013), p. 29.

[39] R. Orús, Advances on tensor network theory: Symmetries, fermions, entanglement, and holography, Eur. Phys. J. B 87, 1 (2014).

[40] T. Nishino, Y. Hieida, K. Okunishi, N. Maeshima, Y. Akutsu, and A. Gendiar, Two-dimensional tensor product variational formulation, Prog. Theor. Phys. 105, 409 (2001).

[41] N. Schuch, D. Poilblanc, J. I. Cirac, and D. Pérez-García, Topological Order in the Projected Entangled-Pair States Formalism: Transfer Operator and Boundary Hamiltonians, Phys. Rev. Lett. 111, 090501 (2013).

[42] D. Poilblanc, J. I. Cirac, and N. Schuch, Chiral topological spin liquids with projected entangled pair states, Phys. Rev. B 91, 224431 (2015).

[43] D. Poilblanc, N. Schuch, and I. Affleck, SU(2) ${ }_{1}$ chiral edge modes of a critical spin liquid, Phys. Rev. B 93, 174414 (2016).

[44] Y. Tang, A. W. Sandvik, and C. L. Henley, Properties of resonating-valence-bond spin liquids and critical dimer models, Phys. Rev. B 84, 174427 (2011).

[45] D. Poilblanc, N. Schuch, D. Pérez-García, and J. I. Cirac, Topological and entanglement properties of resonating valence bond wave functions, Phys. Rev. B 86, 014404 (2012).

[46] N. Schuch, D. Poilblanc, J. I. Cirac, and D. Pérez-García, Resonating valence bond states in the PEPS formalism, Phys. Rev. B 86, 115108 (2012).

[47] P. W. Anderson, Resonating valence bonds: A new kind of insulator? Mater. Res. Bull. 8, 153 (1973).

[48] D. Poilblanc and M. Mambrini, Quantum critical phase with infinite projected entangled paired states, Phys. Rev. B 96, 014414 (2017).

[49] M. Mambrini, R. Orús, and D. Poilblanc, Systematic construction of spin liquids on the square lattice from tensor networks with SU(2) symmetry, Phys. Rev. B 94, 205124 (2016).

[50] T. Nishino and K. Okunishi, Corner transfer matrix renormalization group method, J. Phys. Soc. Jpn. 65, 891 (1996).

[51] D. Poilblanc, Investigation of the chiral antiferromagnetic Heisenberg model using projected entangled pair states, Phys. Rev. B 96, 121118 (2017).

[52] J. Dubail and N. Read, Tensor network trial states for chiral topological phases in two dimensions and a no-go theorem in any dimension, Phys. Rev. B 92, 205307 (2015).

[53] S. R. White, Density Matrix Formulation for Quantum Renormalization Groups, Phys. Rev. Lett. 69, 2863 (1992).

[54] S. Yan, D. A. Huse, and S. R. White, Spin-liquid ground state of the $s=1 / 2$ kagome Heisenberg antiferromagnet, Science 332, 1173 (2011).

[55] Quantum Mechanics, edited by L. D. Landau and E. M. Lifshitz, 3rd ed. (Pergamon, 1977), pp. 354-395.

[56] P. Corboz, Variational optimization with infinite projected entangled-pair states, Phys. Rev. B 94, 035133 (2016).

[57] L. Vanderstraeten, J. Haegeman, P. Corboz, and F. Verstraete, Gradient methods for variational optimization of projected entangled-pair states, Phys. Rev. B 94, 155123 (2016).

[58] M. T. Fishman, L. Vanderstraeten, V. Zauner-Stauber, J. Haegeman, and F. Verstraete, Faster Methods for Contracting Infinite 2D Tensor Networks, arXiv:1711.05881. 
[59] L. Vanderstraeten, M. Mariën, F. Verstraete, and J. Haegeman, Excitations and the tangent space of projected entangled-pair states, Phys. Rev. B 92, 201111 (2015).

[60] N. Regnault and B. A. Bernevig, Fractional Chern Insulator, Phys. Rev. X 1, 021014 (2011).

[61] B. A. Bernevig and N. Regnault, Emergent many-body translational symmetries of Abelian and non-Abelian fractionally filled topological insulators, Phys. Rev. B 85, 075128 (2012).

[62] A. M. Läuchli, Z. Liu, E. J. Bergholtz, and R. Moessner, Hierarchy of Fractional Chern Insulators and Competing Compressible States, Phys. Rev. Lett. 111, 126802 (2013).

[63] H. Li and F. D. M. Haldane, Entanglement Spectrum As a Generalization of Entanglement Entropy: Identification of Topological Order in Non-Abelian Fractional Quantum Hall Effect States, Phys. Rev. Lett. 101, 010504 (2008).

[64] J. I. Cirac, D. Poilblanc, N. Schuch, and F. Verstraete, Entanglement spectrum and boundary theories with projected entangledpair states, Phys. Rev. B 83, 245134 (2011).
[65] S. S. Jahromi, R. Orús, M. Kargarian, and A. Langari, Infinite projected entangled-pair state algorithm for ruby and trianglehoneycomb lattices, Phys. Rev. B 97, 115161 (2018).

[66] F. Pollmann, S. Mukerjee, A. M. Turner, and J. E. Moore, Theory of Finite-Entanglement Scaling at One-Dimensional Quantum Critical Points, Phys. Rev. Lett. 102, 255701 (2009).

[67] J. Haegeman and F. Verstraete, Diagonalizing Transfer Matrices and Matrix Product Operators: A Medley of Exact and Computational Methods, Annu. Rev. Condens. Matter Phys. 8, 355 (2017).

[68] L. Vanderstraeten, J. Haegeman, and F. Verstraete, Tangent-space methods for uniform matrix product states, arXiv: 1810.07006.

[69] C. Gross and I. Bloch, Quantum simulations with ultracold atoms in optical lattices, Science 357, 995 (2017).

[70] A. Hackenbroich, A. Sterdyniak, and N. Schuch, Interplay of SU(2), point group and translation symmetry for PEPS: Application to a chiral spin liquid, Phys. Rev. B 98, 085151 (2018). 